\title{
Relationship between obesity and the occurrence of negative outcome in patients hospitalized with COVID-19: integrative literature review
}

\author{
Relação entre obesidade e a ocorrência de desfecho negativo em pacientes hospitalizados com
}

COVID-19: revisão integrativa da literatura

Relación entre obesidad y ocurrencia de resultado negativo en pacientes hospitalizados con

COVID-19: revisión integradora de la literatura

Received: 09/24/2021 | Reviewed: 10/03/2021 | Accept: 10/05/2021| Published: 10/09/2021

\author{
Eline Fernandes Ribeiro de Castro \\ ORCID: https://orcid.org/0000-0002-6996-7717 \\ Universidade Federal do Pará, Brasil \\ E-mail: fernandeseline@hotmail.com \\ Chriscia Jamilly Pinto de Sousa \\ ORCID: https://orcid.org/0000-0002-6996-7717 \\ Universidade Federal do Pará, Brasil \\ E-mail: jamillysousa@ufpa.br \\ Carolina Heitmann Mares Azevedo Ribeiro \\ ORCID: https://orcid.org/0000-0002-9457-2733 \\ Universidade Federal do Pará, Brasil \\ E-mail: carolmheitmann@ hotmail.com \\ Carlos Augusto Abreu Alberio \\ ORCID: https://orcid.org/0000-0002-9364-3990 \\ Universidade Federal do Pará, Brasil \\ E-mail: alberio@ufpa.br
}

\begin{abstract}
Objective: To verify the relationship between obesity and the occurrence of negative outcomes in hospitalized patients. Methodology: An integrative review was carried out using the National Library of Medicine of the National Institutes of Health (PubMed) and the Virtual Health Library (VLH/BVS) database. Results: It was observed that obese patients are 2 to 5 times more likely to need Invasive Mechanical Ventilation (IMV) when admitted to the Intensive Care Unit. Patients with high BMI (obese) and who needed mechanical ventilation had a mortality rate above $60 \%$. The risk increases as the patient has other pathologies, this fact is shown that mortality by COVID-19 has multifactorial causes. Conclusion: The study showed that obesity is a risk factor associated with the increased development of the severe form of the disease, usually associated with other pathologies (hypertension, diabetes and cardiovascular diseases). That is, obesity increases the likelihood of unfavorable outcomes.
\end{abstract}

Keywords: Obesity; COVID-19; Risk factors.

\section{Resumo}

Objetivo: Verificar a relação entre a obesidade e a ocorrência de desfechos negativos em pacientes hospitalizados. Metodologia: Foi feita uma revisão integrativa, por meio da base de dados Biblioteca Nacional de Medicina dos Institutos Nacionais de Saúde (PubMed) e Biblioteca Virtual em Saúde (VLH/BVS). Resultados: Observou-se que pacientes obesos tem de 2 a 5 vezes mais chances de necessitar de Ventilação Mecânica Invasiva (VMI), quano admitidos em Unidade de Terapia Intensiva. Pacientes que apresentam IMC elevados (obesos) e que precisaram de ventilação mecânica apresentaram mortalidade superior a 60\%. O risco aumenta conforme o paciente apresenta outras patologias, tal fato é mostrado que a mortalidade por COVID-19 tem causas multifatoriais. Conclusão: O estudo mostrou que a obesidade é um fator de risco associado ao aumento do desenvolvimento da forma grave da doença, geralmente associado a outras patologias (hipertensão, diabetes e doenças cardiovasculares). Ou seja, a obesidade aumenta a probabilidade de desfechos desfavoráveis.

Palavras-chave: Obesidade; COVID-19; Fatores de risco.

\section{Resumen}

Objetivo: Verificar la relación entre obesidad y ocurrencia de desenlaces negativos en pacientes hospitalizados. Metodología: Se realizó una revisión integradora utilizando la base de datos de la Biblioteca Nacional de Medicina de los Institutos Nacionales de Salud (PubMed) y la Biblioteca Virtual en Salud (VLH / BVS). Resultados: Se observó que los pacientes obesos tienen de 2 a 5 veces más probabilidades de necesitar Ventilación Mecánica Invasiva (VMI) 
cuando ingresan en la Unidad de Cuidados Intensivos. Los pacientes con IMC alto (obesos) y que necesitaban ventilación mecánica tenían una tasa de mortalidad superior al 60\%. El riesgo aumenta a medida que el paciente resenta otras patologías, este hecho se demuestra que la mortalidad por COVID-19 tiene causas multifactoriales. Conclusión: El estudio mostró que la obesidad es un factor de riesgo asociado al mayor desarrollo de la forma grave de la enfermedad, generalmente asociada a otras patologías (hipertensión, diabetes y enfermedades cardiovasculares). Es decir, la obesidad aumenta la probabilidad de resultados desfavorables.

Palabras clave: Obesidad; COVID-19; Factores de riesgo.

\section{Introduction}

In Wuhan, China, at the end of 2019 was observed a series of cases of a new virus that caused respiratory infections. Since then the SARS-CoV-2 (new coronavirus), according to the data released by World Health Organisation, already infected over 215 millions people around the world, what has already resulted in 4,48 millions of deaths. In Brazil, according to the data released by Brazilian government, it's 578.326 deaths already, reaching a 2.8\% death rate. (OMS, 2021; BRASIL, 2020) The new coronavirus possess an single-stranded RNA enveloped and the transmission is realized human by human, although in China the bats were stablished as an important reservoir of the virus. The most common clinic symptoms are: fever and cough; but can include dyspnea, headache, muscle pain and fatigue. Is estimated that $20 \%$ of the cases are serious, with a $3 \%$ death rate. The COVID-19's complications (respiratory failure, acute respiratory distress syndrome (ARDS), sepsis, septic shock, thromboembolism, and/or multiple organ failure), happens mainly in patients with comorbidities such as hypertension, obesity, diabetes, cardiovascular disease, chronic lung disease, among others. (Hu et al., 2017; Wang, Tang, Wei, 2020; Wang, Horby, Hayden, Gao; 2020)

The (re) emergence of the virus has caused several impacts in many areas of society, such as economy and education, but mostly in the health area. The most critical structural limitation during the pandemic in the country was the low number of ICU beds and mechanical fans needed to deal with the serious cases of the disease, furthermore there were difficulties in the acquisitions of the medicines (sedatives, neuromuscular and cardiotonic blockers), as well as the rising of the prices, with and increase up to $200 \%$ in relation to the usual price. The State University of Campinas estimates that an implantation of an ICU bed is something around $\mathrm{R} \$ 180.000,00$, and the daily cost of it is between R \$ 2.500,00 and R \$ 3.000,00. (UNICAMP, 2020; Paiva, et al,. 2020; Holanda \& Pinheiro, 2020; Saraiva, et al., 2020)

Given that the damages caused to the patient health and the financial impact related to the treatment of serious cases, it's necessary the identification of the predictive risk factors to the development of the severe stage of the disease. Between the factors we can highlight obesity, given that results between other things in a disregulated immune answer, through the increasing of inflamatory cells, for exemple, impacting among other things the pulmonary functions. (Kulcsar et al, 2019; Dixon et al, 2018).

Being the obesity a prevalent desease in the world population, the information about the impact of this risk factor as for the negative outcome of the desease, could be a great value for the directing of the clinic decision, as the rationing of the resources and consequently a reduction of the costs related to this assistance. In this way, the objective of this integrative revision is to verify the relation between obesity and the occurrencies of negative outcome in hospitalized patients.

\section{Methods}

This work is an integrative review based on the research question: "What is the relationship between obesity and the occurrence of negative outcomes in patients hospitalized for COVID-19?”. This was delimited by the PICO strategy (acronym for Patient, Intervention, Comparison, Results). The steps taken in preparing the study were: elaboration of the research 
question, sampling or search in the literature of primary studies, data extraction, evaluation of primary studies, interpretation of results, presentation of the review.

The primary studies were searched from january to 2021, in the following databases: National Library of Medicine of the National Institutes of Health (PubMed) and Virtual Health Library (VLH / BVS).

The controlled descriptors selected in the Health Sciences Descriptors (HSD) of the Virtual Health Library (VLH / BVS) and HSD in PubMed were "Obesity, CoronavirusInfections, Hospitalization, Mortality, Intensive Care Units, Respiration, Artificial" (in English). The descriptors were combined using the Boolean operators, which obtained the following searches: "Obesity AND CoronavirusInfections AND Hospitalization AND Mortality OR Intensive Care Units OR Respiration, Artificial".

The inclusion criteria were articles whose main and / or secondary objective was to verify and/or establish the correlation between obesity and negative outcomes in patients hospitalized with COVID-19. Regarding the period, foram selecionados artigos publicados no ano de 2020. The search was conducted in Portuguese, Spanish and English. The exclusion criteria were: studies carried out in emergency rooms and outpatient services in hospitals, case reports and studies, pregnant and pediatric patients.

The choice of articles was divided into three stages:

1) Search for articles using descriptors in the databases, filtering the period.

2) Reading of titles and abstracts by two independent reviewers who decided to include or exclude the article. In case of disagreement, a third reviewer was invited.

3) Reading of the articles in full by the reviewers, in order to identify whether the article answered the proposed question.

Articles were identified in the four databases. After applying the criteria, 308 articles were excluded, for the following reasons: Duplicates (136), Secondary Studies and / or Case Report (12), No Answer To The Proposed Questions (83), Studies in Outpatients (16) and studies with pregnant women and pediatric patients (22). Thus, 39 articles were qualified for this review.

\section{Results}

The information extracted from the primary studies included in the review is shown in Table 1. 
Research, Society and Development, v. 10, n. 13, e165101321038, 2021

(CC BY 4.0) | ISSN 2525-3409 | DOI: http://dx.doi.org/10.33448/rsd-v10i13.21038

Table 1 - Information extracted from the primary studies.

\begin{tabular}{|c|c|c|c|c|c|c|c|c|c|c|c|c|c|c|}
\hline \multirow{2}{*}{ ID } & \multirow{2}{*}{ Títle } & \multirow{2}{*}{$\begin{array}{l}\text { Author, } \\
\text { Year }\end{array}$} & \multirow{2}{*}{$\begin{array}{l}\text { Language } \\
\text { Country }\end{array}$} & \multirow{2}{*}{ Objective } & \multirow{2}{*}{ Methods } & \multirow{2}{*}{$\mathbf{n}$} & \multirow{2}{*}{ Sex } & \multirow{2}{*}{ Age } & \multicolumn{3}{|c|}{ Obesity } & \multirow{2}{*}{$\begin{array}{c}\text { Statistical } \\
\text { Association with } \\
\text { Obesity }\end{array}$} & \multirow{2}{*}{ Conclusion } & \multirow{2}{*}{ Limitation } \\
\hline & & & & & & & & & $\mathbf{n}$ & Severe & Death & & & \\
\hline 01 & $\begin{array}{l}\text { 30-day mortality in } \\
\text { patients } \\
\text { hospitalized with } \\
\text { COVID-19 during } \\
\text { the first wave of the } \\
\text { Italian epidemic: A } \\
\text { prospective cohort } \\
\text { study. }\end{array}$ & $\begin{array}{l}\text { Giacomelli } \\
\text { et al., } 2020\end{array}$ & $\begin{array}{l}\text { English } \\
\text { Italy }\end{array}$ & $\begin{array}{c}\text { Describe the } \\
\text { demographic and } \\
\text { clinical } \\
\text { characteristics of } \\
\text { hospitalized patients } \\
\text { with COVID-19 and } \\
\text { factors associated } \\
\text { with the risk of death } \\
\text { related to COVID-19. }\end{array}$ & $\begin{array}{l}\text { Prospective } \\
\text { cohort study, } \\
\text { with data } \\
\text { extracted from } \\
\text { the clinical } \\
\text { records of adult } \\
\text { patients. }\end{array}$ & 233 & $\begin{array}{l}161(69,1 \%) \\
\text { Masculine }\end{array}$ & 61 & $38(16,3 \%)$ & - & $\begin{array}{c}25(65,7 \%) \\
25 / 38\end{array}$ & $\begin{array}{c}\text { Mortality } \\
\text { aHR 3,04 [IC 95\% } \\
1,42-6,49]\end{array}$ & $\begin{array}{c}\text { Obesity is } \\
\text { associated with a } 3 \mathrm{x} \\
\text { greater risk of death } \\
\text { than patients with a } \\
\text { BMI }<30 \mathrm{~kg} / \mathrm{m}^{2} .\end{array}$ & $\begin{array}{c}\text { Low number of tests } \\
\text { performed at hospital } \\
\text { admission; } \\
\text { Low number of CT scans } \\
\text { performed; } \\
\text { Difficulty in determining } \\
\text { the effect of medications } \\
\text { on the course of the } \\
\text { disease; }\end{array}$ \\
\hline 02 & $\begin{array}{c}\text { Association of } \\
\text { Body Mass Index } \\
\text { and Age With } \\
\text { Morbidity and } \\
\text { Mortality in } \\
\text { Patients } \\
\text { Hospitalized With } \\
\text { COVID-19: Results } \\
\text { From the American } \\
\text { Heart Association } \\
\text { COVID-19 } \\
\text { Cardiovascular } \\
\text { Disease Registry. }\end{array}$ & $\begin{array}{l}\text { Hendren et } \\
\text { al., } 2021\end{array}$ & $\begin{array}{l}\text { English } \\
\text { USA }\end{array}$ & $\begin{array}{c}\text { Evaluate the } \\
\text { relationship between } \\
\text { body mass index and } \\
\text { mortality, mechanical } \\
\text { ventilation, adverse } \\
\text { cardiovascular, renal } \\
\text { and venous } \\
\text { thromboembolic } \\
\text { outcomes in patients } \\
\text { admitted with } \\
\text { COVID-19 }\end{array}$ & $\begin{array}{l}\text { Retrospective } \\
\text { study, with data } \\
\text { collected from } 88 \\
\text { hospitals in the } \\
\text { USA. }\end{array}$ & 7606 & $\begin{array}{l}4207(55 \%) \\
\text { Masculine }\end{array}$ & 63 & \begin{tabular}{|c|}
3311 \\
$(43,4 \%)$ \\
3311 \\
$(43,5 \%)-$ \\
Obese \\
Class I: \\
1623 \\
$(21,3 \%)$ \\
Class II: \\
$846(111 \%)$ \\
Class III: \\
$842(11 \%)$
\end{tabular} & $\begin{array}{c}\text { VMI } \\
\text { Class I: } 364 \\
\text { (22,5\%); } \\
\text { Class II: } \\
214 \\
(25,3 \%) ; \\
\text { Class III: } \\
223 \\
(26,6 \%) ; \\
(\mathrm{p}<0,001)\end{array}$ & \begin{tabular}{|l} 
Class I: 237 \\
(14,6\%); \\
Class II: \\
132 \\
$(15,6 \%) ;$ \\
Class III: \\
$223(26,5 \%)$ \\
$(\mathrm{p}<0,001)$
\end{tabular} & \begin{tabular}{|} 
Mortality \\
Class III (HRR, 1.30 \\
[95\% CI, 1.03-1.63]); \\
VMI \\
Class I (OR $=1,54$ [IC \\
95\%: 1,29-1,84]); \\
Class II (OR $=1,88$ \\
[95\% CI: $1,52-2,32]) ;$ \\
Class III (OR $=2,08$ \\
[95\% CI: $1,68-2,58]) ;$
\end{tabular} & $\begin{array}{l}\text { Obesity is } \\
\text { associated with } \\
\text { increased risk of } \\
\text { death, mechanical } \\
\text { ventilation, } \\
\text { occurrence of } \\
\text { thromboembolism } \\
\text { and need for } \\
\text { dialysis, especially } \\
\text { in younger patients. }\end{array}$ & $\begin{array}{l}\text { The non-generalization of } \\
\text { data to smaller hospitals. } \\
\text { Information may have been } \\
\text { underreported. The } \\
\text { researchers were unable to } \\
\text { assess whether the } \\
\text { occurrence of no } \\
\text { resuscitation order was a } \\
\text { factor that interfered with } \\
\text { the established primary } \\
\text { outcome. }\end{array}$ \\
\hline 03 & $\begin{array}{c}\text { BMI as a Risk } \\
\text { Factor for Clinical } \\
\text { Outcomes in } \\
\text { Patients } \\
\text { Hospitalized with } \\
\text { COVID-19 in New } \\
\text { York. }\end{array}$ & $\begin{array}{c}\text { Kim et al., } \\
2020\end{array}$ & $\begin{array}{l}\text { English } \\
\text { USA }\end{array}$ & $\begin{array}{l}\text { Study the association } \\
\text { between BMI and } \\
\text { clinical outcomes } \\
\text { among patients with } \\
\text { coronavirus infection } \\
\text { in } 2019 \text { (COVID-19) }\end{array}$ & $\begin{array}{c}\text { Retrospective } \\
\text { study analyzing } \\
\text { electronic } \\
\text { medical records } \\
\text { of patients } \\
\text { diagnosed with } \\
\text { Covid-19 } \\
\text { admitted to } 12 \\
\text { hospitals in the } \\
\text { USA. }\end{array}$ & 10.861 & $\begin{array}{c}6468 \\
(59,6 \%) \\
\text { Masculine }\end{array}$ & 65 & $\begin{array}{c}4090 \\
\text { (37,7\%) - } \\
\text { Obese } \\
\text { Class I: } \\
2341 \\
(21,6 \%) \\
\text { Class II: } \\
990(9,1 \%) \\
\text { Class III: } \\
755(7,0 \%)\end{array}$ & $\begin{array}{c}\text { IMV } \\
\text { Class I: } 517 \\
\text { (22,1\%); } \\
\text { Class II: } \\
248 \\
(25,1 \%) ; \\
\text { Class III: } \\
200 \\
(26,6 \%) ; \\
(\mathrm{p}<0,001)\end{array}$ & $\begin{array}{c}\text { Class I: } 470 \\
\text { (20,1\%); } \\
\text { Class II: } \\
207 \\
(20,9 \%) ; \\
\text { Class III: } \\
165(21,9 \%)\end{array}$ & \begin{tabular}{|c} 
BMI: \\
Class I (OR $=1,48$ [IC \\
95\%: $1,27-1,72]$; \\
Class II (OR $=1,89$ \\
[95 \% CI: $1,56-2,28]) ;$ \\
Class III (OR $=2,31$ \\
[95\% CI: $1,88-2,85]) ;$ \\
Mortality \\
Class II: OR $=1,25$ \\
[IC 95\%: $1,03-1,52]$ \\
Class III: OR $=1,61$ \\
[IC 95\%: $1,30-2,00]$
\end{tabular} & $\begin{array}{c}\text { All classes of } \\
\text { obesity were } \\
\text { associated with } \\
\text { increased risk of } \\
\text { IMV. In addition, } \\
\text { grade II obesity and } \\
\text { grade III obesity } \\
\text { were associated } \\
\text { with greater } \\
\text { chances of death. } \\
\text { However, among } \\
\text { intubated patients, } \\
\text { BMI was not } \\
\text { statistically } \\
\text { associated with the } \\
\text { risk of death. } \\
\end{array}$ & $\begin{array}{l}\text { Use of Administrative data } \\
\text { that may limit clinical } \\
\text { information; } \\
\text { Difference between patient } \\
\text { populations; }\end{array}$ \\
\hline 04 & $\begin{array}{c}\text { Clinical } \\
\text { characteristics of } \\
\text { coronavirus disease } \\
2019 \text { in a single } \\
\text { center of Argentina. } \\
\text { Retrospective } \\
\text { cohort }\end{array}$ & $\begin{array}{l}\text { Castro et } \\
\text { al., } 2020\end{array}$ & $\begin{array}{l}\text { English } \\
\text { Argentina }\end{array}$ & $\begin{array}{l}\text { Evaluate the clinical } \\
\text { characteristics, } \\
\text { evolution and } \\
\text { severity of COVID- } \\
19 \text { in a third-level } \\
\text { hospital in Argentina }\end{array}$ & $\begin{array}{c}\text { Retrospective } \\
\text { cohort of } 101 \\
\text { patients with } \\
\text { COVID-19. } \\
\text { Patients were } \\
\text { divided } \\
\text { according to the } \\
\text { presence or } \\
\text { absence of } \\
\end{array}$ & 101 & $\begin{array}{l}54(53 \%) \\
\text { Feminine }\end{array}$ & 42 & $18(18 \%)$ & $\begin{array}{c}\text { Pneumonia } \\
8(31 \%) \\
(8 / 26)\end{array}$ & - & $\begin{array}{l}\text { Severe Pneumonia } \\
\quad \mathrm{p}(0,061)\end{array}$ & $\begin{array}{l}\text { There was no } \\
\text { significant } \\
\text { correlation between } \\
\text { obesity and the } \\
\text { occurrence of } \\
\text { severe pneumonia }\end{array}$ & $\begin{array}{l}\text { Retrospective analysis; } \\
\text { Few laboratory and } \\
\text { radiological tests; } \\
\text { Limited study population; }\end{array}$ \\
\hline
\end{tabular}


Research, Society and Development, v. 10, n. 13, e165101321038, 2021

(CC BY 4.0) | ISSN 2525-3409 | DOI: http://dx.doi.org/10.33448/rsd-v10i13.21038

\begin{tabular}{|c|c|c|c|c|c|c|c|c|c|c|c|c|c|c|}
\hline & & & & & $\begin{array}{l}\text { pneumonia and } \\
\text { disease severity. }\end{array}$ & & & & & & & & & \\
\hline 05 & $\begin{array}{l}\text { Characteristics and } \\
\text { Risk factors for } \\
\text { mortality in patients } \\
\text { hospitalized by } \\
\text { COVID-19 in a } \\
\text { public hospital in } \\
\text { Tacna. }\end{array}$ & $\begin{array}{l}\text { Zavaleta et } \\
\text { al., } 2021\end{array}$ & $\begin{array}{l}\text { Spanish } \\
\text { Peru }\end{array}$ & $\begin{array}{l}\text { Describe the clinical, } \\
\text { laboratory and } \\
\text { treatment } \\
\text { characteristics of } \\
\text { patients hospitalized } \\
\text { for COVID-19 and } \\
\text { determine the risk } \\
\text { factors for in-hospital } \\
\text { mortality. }\end{array}$ & $\begin{array}{c}\text { Retrospective } \\
\text { cohort of adult } \\
\text { patients } \\
\text { hospitalized by } \\
\text { COVID-19. } \\
\text { Clinical, } \\
\text { laboratory and } \\
\text { treatment data } \\
\text { were extracted } \\
\text { from the medical } \\
\text { records of } \\
\text { hospitalized } \\
\text { patients }\end{array}$ & 351 & $\begin{array}{c}260(74,1 \%) \\
\text { Masculine }\end{array}$ & 61 & $111(31,6 \%)$ & - & $\begin{array}{c}42(37,8 \%) \\
(42 / 111)\end{array}$ & - & $\begin{array}{c}\text { There was no } \\
\text { significant } \\
\text { correlation between } \\
\text { obesity and the } \\
\text { occurrence of } \\
\text { pneumonia. }\end{array}$ & $\begin{array}{l}\text { Retrospective analysis; } \\
\text { Limited study population; }\end{array}$ \\
\hline 06 & $\begin{array}{c}\text { Characteristics, } \\
\text { predictors and } \\
\text { outcomes among } 99 \\
\text { patients } \\
\text { hospitalized with } \\
\text { COVID-19 in a } \\
\text { tertiary care center } \\
\text { in Switzerland: an } \\
\text { observational } \\
\text { analysis }\end{array}$ & $\begin{array}{l}\text { Gregoriano } \\
\text { et al., } 2020\end{array}$ & $\begin{array}{l}\text { English } \\
\text { Switzerland }\end{array}$ & $\begin{array}{c}\text { Describe the } \\
\text { admission } \\
\text { characteristics, risk } \\
\text { factors and outcomes } \\
\text { of patients with } 2019 \\
\text { coronavirus disease } \\
\text { (COVID-19) } \\
\text { hospitalized in a } \\
\text { tertiary hospital in } \\
\text { Switzerland }\end{array}$ & $\begin{array}{l}\text { Retrospective } \\
\text { cohort study } \\
\text { included adult } \\
\text { patients with } \\
\text { (SARS-CoV-2) } \\
\text { infection. }\end{array}$ & 99 & $\begin{array}{l}63(62,5 \%) \\
\text { Masculine }\end{array}$ & 67 & $27(27 \%)$ & $\begin{array}{l}\text { Severe } \\
12(34 \%) \\
(12 / 35)\end{array}$ & - & $\begin{array}{c}\text { Severe } \\
\text { OR } 1,70(\mathrm{IC}=0,69- \\
4,22) \\
\mathrm{p}=0,249\end{array}$ & $\begin{array}{l}\text { There was no } \\
\text { significant } \\
\text { correlation between } \\
\text { obesity and the } \\
\text { outcome assessed. }\end{array}$ & $\begin{array}{l}\text { Absence of data in medical } \\
\text { records; } \\
\text { Patients transferred during } \\
\text { hospitalization; } \\
\text { retrospective analysis; }\end{array}$ \\
\hline 07 & $\begin{array}{c}\text { Clinical } \\
\text { characteristics and } \\
\text { day-90 outcomes of } \\
4244 \text { critically ill } \\
\text { adults with } \\
\text { COVID-19: a } \\
\text { prospective cohort } \\
\text { study }\end{array}$ & $\begin{array}{l}\text { COVID- } \\
\text { ICU Group } \\
\text { on behalf } \\
\text { of the } \\
\text { REVA } \\
\text { Network } \\
\text { and the } \\
\text { COVID- } \\
\text { ICU } \\
\text { Investigato } \\
\text { rs* }\end{array}$ & $\begin{array}{l}\text { English } \\
\text { France, } \\
\text { Belgium e } \\
\text { Switzerland }\end{array}$ & $\begin{array}{l}\text { Describe the severity } \\
\text { of acute respiratory } \\
\text { distress syndrome } \\
\text { (ARDS), the } \\
\text { management of } \\
\text { ventilation, and the } \\
\text { outcomes of ICU } \\
\text { patients with } \\
\text { laboratory-confirmed } \\
\text { COVID-19, and } \\
\text { determine risk factors } \\
\text { for mortality within } \\
90 \text { days of admission } \\
\text { to the ICU. }\end{array}$ & $\begin{array}{c}\text { Multicenter } \\
\text { prospective } \\
\text { cohort conducted } \\
\text { in } 138 \text { hospitals } \\
\text { in France, } \\
\text { Belgium and } \\
\text { Switzerland. } \\
\text { Demographic, } \\
\text { clinical, } \\
\text { respiratory } \\
\text { support, adjuvant } \\
\text { interventions, } \\
\text { length of stay in } \\
\text { the ICU and } \\
\text { survival were } \\
\text { collected. }\end{array}$ & 4.244 & $\begin{array}{c}3159 \\
(74,4 \%) \\
\text { Masculine }\end{array}$ & 63 & $1607(41 \%)$ & - & $\begin{array}{c}440(27,3 \%) \\
440 / 1607\end{array}$ & - & $\begin{array}{l}\text { Mortality was } \\
\text { higher in elderly, } \\
\text { diabetic, obese and } \\
\text { severe ARDS } \\
\text { patients. }\end{array}$ & $\begin{array}{l}\text { Absence of data; } \\
\text { Lack of standardization in } \\
\text { data collection }\end{array}$ \\
\hline 08 & $\begin{array}{c}\text { Clinical } \\
\text { characteristics and } \\
\text { in-hospital } \\
\text { mortality of } \\
\text { COVID-19 adult } \\
\text { patients in Saudi } \\
\text { Arabia }\end{array}$ & $\begin{array}{l}\text { Abohamr } \\
\text { et al., } 2020\end{array}$ & $\begin{array}{c}\text { English } \\
\text { Saudi Arabia }\end{array}$ & $\begin{array}{l}\text { Provide a detailed } \\
\text { study of } \\
\text { demographic, } \\
\text { baseline } \\
\text { comorbidities, } \\
\text { clinical features, and } \\
\text { outcomes for patients } \\
\text { with coronavirus } \\
\text { disease in 2019 } \\
\text { (COVID-19). }\end{array}$ & $\begin{array}{l}\text { Study of case } \\
\text { series, of } \\
\text { inpatient } \\
\text { registration. }\end{array}$ & 768 & $\begin{array}{l}589(76,7 \%) \\
\text { Masculine }\end{array}$ & $\cong 46$ & $\begin{array}{c}143 \\
(18 \quad 6 \%)\end{array}$ & $\begin{array}{c}\text { ICU } \\
102(71,3 \%)\end{array}$ & $\begin{array}{l}63(44 \%) \\
(63 / 143)\end{array}$ & $\begin{array}{c}\mathrm{ICU} \\
\mathrm{OR}=3,732[\mathrm{IC}= \\
2.511-5.546]\end{array}$ & $\begin{array}{c}\text { There was a } \\
\text { statistical } \\
\text { association between } \\
\text { obesity, ICU } \\
\text { admission and } \\
\text { death. }\end{array}$ & $\begin{array}{l}\text { Limited study population; } \\
\text { It was difficult to obtain } \\
\text { data on admission; }\end{array}$ \\
\hline
\end{tabular}


Research, Society and Development, v. 10, n. 13, e165101321038, 2021

(CC BY 4.0) | ISSN 2525-3409 | DOI: http://dx.doi.org/10.33448/rsd-v10i13.21038

\begin{tabular}{|c|c|c|c|c|c|c|c|c|c|c|c|c|c|c|}
\hline 09 & $\begin{array}{c}\text { Clinical } \\
\text { characteristics and } \\
\text { outcomes in women } \\
\text { and men } \\
\text { hospitalized for } \\
\text { coronavirus disease } \\
2019 \text { in New } \\
\text { Orleans. }\end{array}$ & $\begin{array}{l}\text { Yoshida et } \\
\text { al., } 2021\end{array}$ & $\begin{array}{l}\text { English } \\
\text { USA }\end{array}$ & $\begin{array}{c}\text { Determine if sex } \\
\text { differences exist in } \\
\text { clinical } \\
\text { characteristics and } \\
\text { outcomes of adults } \\
\text { hospitalized for } \\
\text { coronavirus disease } \\
\text { 2019 (COVID-19) in } \\
\text { a US healthcare } \\
\text { system. }\end{array}$ & $\begin{array}{c}\text { Demographic } \\
\text { data, } \\
\text { comorbidities, } \\
\text { presenting } \\
\text { symptoms and } \\
\text { laboratory } \\
\text { results. } \\
\text { Outcomes } \\
\text { included } \\
\text { admission to the } \\
\text { intensive care } \\
\text { unit (ICU), } \\
\text { invasive } \\
\text { mechanical } \\
\text { ventilation } \\
\text { (IMV), and } \\
\text { hospital death. }\end{array}$ & 776 & $\begin{array}{c}476(61,4 \%) \\
\text { Women }\end{array}$ & $\cong 60$ & $411 \quad(53 \%)$ & - & - & $\begin{array}{c}\text { ICU } \\
\text { Obesity: OR } 1.55 \text { [IC } \\
\text { 1.10-2.17] } \\
\text { BMI >40: OR 2.48 } \\
\text { [IC 1.43-4.29] } \\
\text { VMI } \\
\text { Obesity: OR } 2.41 \\
\text { [IC:1.63-3.56] } \\
\text { BMI 35-39.9: OR } \\
2.68 \text { [IC 1.42-5.06]; } \\
\text { BMI >40: OR 3.85 } \\
\text { [IC:2.07-7.17] }\end{array}$ & $\begin{array}{c}\text { Obesity was } \\
\text { associated with } \\
\text { Mechanical } \\
\text { Ventilation at a } \\
\text { lower BMI (> 35) } \\
\text { in women, but the } \\
\text { effect of morbid } \\
\text { obesity (BMI } \geq 40 \text { ) } \\
\text { was similar in both } \\
\text { sexes. When } \\
\text { multivariate } \\
\text { analyzes were } \\
\text { performed by race, } \\
\text { obesity was } \\
\text { associated with } \\
\text { greater odds of } \\
\text { ICU, IMV and } \\
\text { death in black } \\
\text { patients in general. } \\
\text { However, obesity } \\
\text { was not associated } \\
\text { with death in non- } \\
\text { black women and } \\
\text { men. }\end{array}$ & $\begin{array}{l}\text { Limited population; } \\
\text { Limited number of patients } \\
\text { from some ethnic groups, } \\
\text { such as Asians and Native } \\
\text { Americans, preventing } \\
\text { more accurate racial/ethnic } \\
\text { stratification in the } \\
\text { analysis. }\end{array}$ \\
\hline 10 & $\begin{array}{c}\text { Clinical } \\
\text { characteristics and } \\
\text { outcomes of } \\
\text { critically ill patients } \\
\text { with COVID-19 } \\
\text { admitted to an } \\
\text { intensive care unit } \\
\text { in London: A } \\
\text { prospective } \\
\text { observational } \\
\text { cohort study }\end{array}$ & $\begin{array}{l}\text { Thomson } \\
\text { et al., } 2020\end{array}$ & $\begin{array}{l}\text { English } \\
\text { United } \\
\text { Kingdom }\end{array}$ & $\begin{array}{l}\text { Understand the } \\
\text { clinical } \\
\text { characteristics and } \\
\text { outcomes of patients } \\
\text { admitted to a United } \\
\text { Kingdom ICU with } \\
\text { severe COVID-19. }\end{array}$ & $\begin{array}{c}\text { Prospective } \\
\text { observational } \\
\text { cohort of all } \\
\text { patients with } \\
\text { COVID-19 } \\
\text { admitted to a } \\
\text { large United } \\
\text { Kingdom ICU. }\end{array}$ & 156 & $\begin{array}{l}112(72 \%) \\
\text { Masculine }\end{array}$ & 62 & $31(20,1 \%)$ & - & - & $\begin{array}{c}\text { Death } \\
\text { OR 3,06 [IC 95\% } \\
\text { 1,16-8,74]; }\end{array}$ & $\begin{array}{l}\text { Obese patients in } \\
\text { the ICU were } 3 \\
\text { (three) times more } \\
\text { likely to evolve to } \\
\text { death. }\end{array}$ & $\begin{array}{l}\text { The screening and criteria } \\
\text { used to identify patients } \\
\text { who require (and are } \\
\text { suitable for) admission to } \\
\text { the ICU may have affected } \\
\text { our cohort composition } \\
\text { and, potentially, } \\
\text { relationships between } \\
\text { exposures and outcomes. }\end{array}$ \\
\hline 11 & $\begin{array}{c}\text { Clinical } \\
\text { Characteristics and } \\
\text { Outcomes of } \\
\text { Patients } \\
\text { Hospitalized for } \\
\text { COVID-19 in } \\
\text { Africa: Early } \\
\text { Insights from the } \\
\text { Democratic } \\
\text { Republic of the } \\
\text { Congo } \\
\end{array}$ & $\begin{array}{l}\text { Nachega et } \\
\text { al., } 2020\end{array}$ & $\begin{array}{l}\text { English } \\
\text { Congo } \\
\text { Republic }\end{array}$ & $\begin{array}{l}\text { Describe the clinical, } \\
\text { laboratory and } \\
\text { outcome } \\
\text { characteristics of } \\
\text { patients hospitalized } \\
\text { with COVID-19 in } \\
\text { the DRC and } \\
\text { differentiate them } \\
\text { from other non- } \\
\text { African populations. }\end{array}$ & $\begin{array}{c}\text { Cohort using } \\
\text { data routinely } \\
\text { collected from } \\
\text { the DRC } \\
\text { Ministry of } \\
\text { Health } \\
\text { Multisectoral } \\
\text { Response } \\
\text { Committee } \\
\text { database } \\
\text { COVID-19 } \\
\end{array}$ & 766 & $\begin{array}{c}500(65,3 \%) \\
\text { Masculine }\end{array}$ & 46 & $39(5,1 \%)$ & $\begin{array}{l}\text { Serious } \\
22(56,4 \%) \\
(22 / 39)\end{array}$ & $\begin{array}{c}17(43 \%) \\
(17 / 39)\end{array}$ & $\begin{array}{c}\text { Death } \\
\text { aHR } 2,30 \text { [ IC } 95 \% \\
1,24-4,27]\end{array}$ & $\begin{array}{l}\text { Obesity increases } \\
\text { the risk of death by } \\
2 \text { (two) times. }\end{array}$ & $\begin{array}{l}\text { It was not possible to } \\
\text { compare clinical } \\
\text { characteristics between } \\
\text { hospitalized patients with } \\
\text { COVID-19 and outpatients. }\end{array}$ \\
\hline 12 & $\begin{array}{c}\text { Clinical } \\
\text { Characteristics and } \\
\text { Prognosis of } 244 \\
\text { Cardiovascular } \\
\text { Patients Suffering } \\
\text { From Coronavirus } \\
\text { Disease in Wuhan, } \\
\text { China. }\end{array}$ & $\begin{array}{l}\text { Peng, et } \\
\text { al., } 2020\end{array}$ & $\begin{array}{l}\text { English } \\
\text { China }\end{array}$ & $\begin{array}{c}\text { Summarize the } \\
\text { clinical } \\
\text { characteristics and } \\
\text { laboratory indicators } \\
\text { of patients with } \\
\text { COVID - } 19 \text { with } \\
\text { CVD, especially } \\
\text { critically ill patients. }\end{array}$ & $\begin{array}{l}\text { Clinical, } \\
\text { laboratory and } \\
\text { outcome data } \\
\text { were collected } \\
\text { from the patients' } \\
\text { medical records } \\
\text { and compared } \\
\text { between the 2 } \\
\end{array}$ & 244 & $\begin{array}{c}131 \\
(53,69 \%) \\
\text { Feminine }\end{array}$ & 61 & $\begin{array}{l}80(32,7 \%) \\
\text { BMI } \geq 24\end{array}$ & - & $\begin{array}{l}34(42,5 \%) \\
\quad(34 / 80)\end{array}$ & $\begin{array}{c}\text { Death } \\
\text { Obesity: OR 8,43 [IC } \\
95 \% 3,81-18,64]\end{array}$ & $\begin{array}{l}\text { Overweight/obesity } \\
\text { combined with } \\
\text { coronary heart } \\
\text { disease, severe } \\
\text { hypoxia and lactic } \\
\text { acid accumulation } \\
\text { resulting from } \\
\text { respiratory failure }\end{array}$ & $\begin{array}{l}\text { Descriptive study; } \\
\text { Limited number of cases; }\end{array}$ \\
\hline
\end{tabular}


Research, Society and Development, v. 10, n. 13, e165101321038, 2021

(CC BY 4.0) | ISSN 2525-3409 | DOI: http://dx.doi.org/10.33448/rsd-v10i13.21038

\begin{tabular}{|c|c|c|c|c|c|c|c|c|c|c|c|c|c|c|}
\hline & & & & & groups & & & & & & & & $\begin{array}{l}\text { are related to } \\
\text { unfavorable } \\
\text { outcomes. }\end{array}$ & \\
\hline 13 & $\begin{array}{c}\text { Clinical } \\
\text { characteristics and } \\
\text { risk factors } \\
\text { associated with } \\
\text { severe COVID-19: } \\
\text { prospective analysis } \\
\text { of } 1,045 \\
\text { hospitalized cases } \\
\text { in North-Eastern } \\
\text { France, March } \\
2020 .\end{array}$ & $\begin{array}{l}\text { Kaeuffer et } \\
\text { al., } 2020\end{array}$ & $\begin{array}{l}\text { English } \\
\text { France }\end{array}$ & $\begin{array}{l}\text { Identify predictive } \\
\text { risk factors for } \\
\text { serious illness and } \\
\text { death in France }\end{array}$ & $\begin{array}{c}\text { Prospective non- } \\
\text { interventional } \\
\text { study of } \\
\text { hospitalized } \\
\text { COVII-19 adult } \\
\text { patients. Severe } \\
\text { illness was } \\
\text { defined by } \\
\text { composite } \\
\text { criteria, } \\
\text { including death } \\
\text { or admission to } \\
\text { the ICU within } 7 \\
\text { days of } \\
\text { hospitalization }\end{array}$ & 1045 & $\begin{array}{c}612(58,6 \%) \\
\text { Masculine }\end{array}$ & 66 & $\begin{array}{c}351(39,1 \%) \\
(351 / 897)\end{array}$ & $\begin{array}{c}\text { Serious } \\
164(46,7 \%) \\
(164 / 351)\end{array}$ & - & $\begin{array}{l}\text { Serious illness } \\
\text { Obesity: OR 2,2 [IC } \\
\text { 95\% 1,5-3,3]; } \\
\text { Death } \\
\text { Obesity: OR 1,4 [IC } \\
\text { 95\% 0,7 - 2,5] }\end{array}$ & $\begin{array}{l}\text { Obesity is a risk } \\
\text { factor for severe } \\
\text { COVID-19, but it is } \\
\text { not related to the } \\
\text { death of } \\
\text { hospitalized } \\
\text { patients. }\end{array}$ & $\begin{array}{l}\text { Possible lack of data; } \\
\text { self-report of } \\
\text { comorbidities; } \\
\text { There were no d-dimer } \\
\text { tests and/or other } \\
\text { inflammatory markers. }\end{array}$ \\
\hline 14 & $\begin{array}{l}\text { Clinical outcomes } \\
\text { and inflammatory } \\
\text { marker levels in } \\
\text { patients with } \\
\text { Covid-19 and } \\
\text { obesity at an inner- } \\
\text { city safety net } \\
\text { hospital. }\end{array}$ & $\begin{array}{l}\text { Mostaghim } \\
\text {, et al., } \\
2020\end{array}$ & $\begin{array}{l}\text { English } \\
\text { USA }\end{array}$ & $\begin{array}{l}\text { Characterize the } \\
\text { association between } \\
\text { clinical outcomes in } \\
\text { patients with obesity } \\
\text { and inflammatory } \\
\text { markers }\end{array}$ & $\begin{array}{c}\text { Retrospective } \\
\text { cohort where } \\
\text { there was a } \\
\text { comparison } \\
\text { between } \\
\text { outcomes and } \\
\text { inflammatory } \\
\text { markers in } \\
\text { patients with } \\
\text { Covid-19 } \\
\text { stratified by BMI } \\
\text { and T2D. } \\
\end{array}$ & 791 & $\begin{array}{c}460(58,2 \%) \\
\text { Masculine }\end{array}$ & 65 & $363(45,9 \%)$ & $\begin{array}{c}\text { ICU } \\
98(52,4 \%)\end{array}$ & & $\begin{array}{c}\text { Death } \\
\text { BMI } \geq 35: \text { aOR }=4,27 \\
\text { [IC 95\% 1,69-10,82) }\end{array}$ & $\begin{array}{l}\text { Obese patients had } \\
\text { a higher transfer } \\
\text { rate to the ICU } \\
(27.1 \% \text { vs } 20.7 \%, p \\
=0.04) \text {. BMI } \geq 35 \\
\text { was associated with } \\
\text { a two-fold greater } \\
\text { risk of transfer to } \\
\text { the ICU and a four- } \\
\text { fold greater risk of } \\
\text { all-cause mortality; }\end{array}$ & $\begin{array}{l}\text { Limited data, observational } \\
\text { study }\end{array}$ \\
\hline 15 & $\begin{array}{l}\text { Comorbidities and } \\
\text { Mortality in } \\
\text { Patients With } \\
\text { COVID-19 Aged } \\
60 \text { Years and Older } \\
\text { in a University } \\
\text { Hospital in Spain. }\end{array}$ & $\begin{array}{l}\text { Posso et } \\
\text { al., } 2020\end{array}$ & $\begin{array}{l}\text { English } \\
\text { Spanish }\end{array}$ & $\begin{array}{l}\text { To analyze whether } \\
\text { the type of } \\
\text { comorbidities } \\
\text { increases the risk of } \\
\text { in-hospital mortality } \\
\text { in patients with } \\
\text { COVID-19 aged } 60 \\
\text { years or more treated } \\
\text { at the university } \\
\text { hospital }\end{array}$ & $\begin{array}{l}\text { Retrospective } \\
\text { evaluation of } \\
\text { prospectively } \\
\text { collected data } \\
\text { from clinical } \\
\quad \text { records }\end{array}$ & 834 & $\begin{array}{c}446(53,5 \%) \\
\text { Feminine }\end{array}$ & $\cong 72$ & $55(6,6 \%)$ & - & $\begin{array}{c}12(21,8 \%) \\
(12 / 55)\end{array}$ & $\begin{array}{c}\text { Death } \\
\text { aOR } 1,21[\mathrm{CI} 95 \% \\
0,60-2,45) \\
\mathrm{p}>0,05\end{array}$ & $\begin{array}{l}\text { There was a higher } \\
\text { risk of obese } \\
\text { patients evolving to } \\
\text { death, but the } \\
\text { correlation was not } \\
\text { significant. }\end{array}$ & $\begin{array}{l}\text { Limited number of cases; } \\
\text { Absence of information in } \\
\text { medical records; } \\
\text { It was not possible to } \\
\text { establish the effect of } \\
\text { treatment on the outcome; }\end{array}$ \\
\hline 16 & 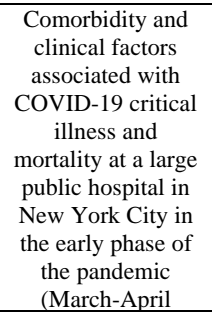 & $\begin{array}{l}\text { Filardo et } \\
\text { al., } 2020\end{array}$ & $\begin{array}{l}\text { English } \\
\text { USA }\end{array}$ & $\begin{array}{c}\text { Evaluate } \\
\text { demographic data, } \\
\text { comorbid conditions } \\
\text { and clinical factors } \\
\text { associated with } \\
\text { critical illness and } \\
\text { mortality among } \\
\text { patients diagnosed } \\
\text { with COVID-19 }\end{array}$ & $\begin{array}{l}\text { Retrospective } \\
\text { study of patients } \\
\text { diagnosed with } \\
\text { COVID-19 }\end{array}$ & 337 & $\begin{array}{c}182(67,4 \%) \\
\text { Masculine }\end{array}$ & 58 & $\begin{array}{c}109(41,8 \% \\
(109 / 261)\end{array}$ & $\begin{array}{c}\text { ICU } \\
59(54,1 \%) \\
(59 / 109)\end{array}$ & $\begin{array}{c}35(32,1 \%) \\
(35 / 109)\end{array}$ & $\begin{array}{c}\text { Mortality } \\
\text { (aRR 1,37, 95\% CI } \\
1,07-1,74)\end{array}$ & $\begin{array}{c}\text { There was a } \\
\text { statistical } \\
\text { association between } \\
\text { obesity and } \\
\text { increased mortality. }\end{array}$ & $\begin{array}{l}\text { Retrospective analysis (no } \\
\text { data) }\end{array}$ \\
\hline
\end{tabular}


Research, Society and Development, v. 10, n. 13, e165101321038, 2021

(CC BY 4.0) | ISSN 2525-3409 | DOI: http://dx.doi.org/10.33448/rsd-v10i13.21038

\begin{tabular}{|c|c|c|c|c|c|c|c|c|c|c|c|c|c|c|}
\hline & 2020). & & & & & & & & & & & & & \\
\hline 17 & $\begin{array}{l}\text { COVID-19 } \\
\text { Experience in a } \\
\text { Wisconsin } \\
\text { Academic Medical } \\
\text { Center. }\end{array}$ & $\begin{array}{c}\text { Kolinski et } \\
\text { al., } 2020\end{array}$ & $\begin{array}{l}\text { English } \\
\text { USA }\end{array}$ & $\begin{array}{l}\text { Describe the } \\
\text { demographic } \\
\text { characteristics and } \\
\text { outcomes of patients } \\
\text { admitted with } \\
\text { COVID-19. }\end{array}$ & 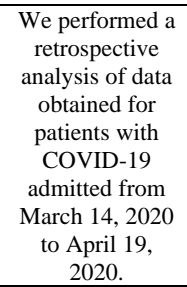 & 178 & $\begin{array}{l}93(55 \%) \\
\text { Masculine }\end{array}$ & 65 & $\begin{array}{l}80(48 \%) \\
(80 / 168)\end{array}$ & $\begin{array}{c}\mathrm{ICU} \\
50(62,5 \%) \\
(50 / 80)\end{array}$ & - & $\begin{array}{c}\text { ICU } \\
\mathrm{p}=0,021\end{array}$ & $\begin{array}{l}\text { There was a } \\
\text { statistical } \\
\text { association between } \\
\text { obesity and } \\
\text { admission to the } \\
\text { ICU. }\end{array}$ & \\
\hline 18 & $\begin{array}{l}\text { COVID-19 } \\
\text { hospitalizations in } \\
\text { Brazil's Unified } \\
\text { Health System } \\
\text { (SUS). }\end{array}$ & $\begin{array}{c}\text { De } \\
\text { Andrade et } \\
\text { al., } 2020\end{array}$ & $\begin{array}{c}\text { English } \\
\text { Brazil }\end{array}$ & $\begin{array}{l}\text { Understand the } \\
\text { profile of hospital } \\
\text { admissions of } \\
\text { COVID-19 in the } \\
\text { Unified Health } \\
\text { System (SUS) and } \\
\text { identify the factors } \\
\text { associated with the } \\
\text { occurrence of } \\
\text { hospital deaths } \\
\text { related to the disease, } \\
\text { considering the } \\
\text { characteristics of the } \\
\text { patient and the care } \\
\text { provided. focusing on } \\
\text { regional variations. }\end{array}$ & $\begin{array}{l}\text { Analysis of } \\
\text { medical records } \\
\text { of hospitalized } \\
\text { patients over } 18 \\
\text { years of age in } \\
\text { the SUS }\end{array}$ & 89.450 & $\begin{array}{c}50539 \\
(56,5 \%) \\
\text { Masculine }\end{array}$ & 60 & $665(0,7 \%)$ & - & $\begin{array}{c}224(33 \%) \\
224 / 665\end{array}$ & $\begin{array}{c}\text { Mortality } \\
\text { OR: } 1,8 \text { [IC } 95 \% 1.5- \\
2,25] \\
\mathrm{p}<0.0001\end{array}$ & $\begin{array}{l}\text { Obesity was } \\
\text { statistically } \\
\text { associated with a } \\
\text { greater chance of } \\
\text { hospital death } \\
(56.3 \% \text { higher } \\
\text { among obese } \\
\text { people compared to } \\
\text { non-obese people). }\end{array}$ & $\begin{array}{l}\text { High underreporting; } \\
\text { Data from the public } \\
\text { network only; }\end{array}$ \\
\hline 19 & $\begin{array}{l}\text { Demographic and } \\
\text { clinical features of } \\
\text { critically ill patients } \\
\text { with COVID-19 in } \\
\text { Greece: The burden } \\
\text { of diabetes and } \\
\text { obesity. }\end{array}$ & $\begin{array}{l}\text { Halvatsioti } \\
\text { s, et al., } \\
\quad 2020\end{array}$ & $\begin{array}{l}\text { English } \\
\text { Greece }\end{array}$ & $\begin{array}{l}\text { To investigate the } \\
\text { association between } \\
\text { type } 2 \text { diabetes } \\
\text { mellitus, other } \\
\text { underlying diseases } \\
\text { and obesity with the } \\
\text { outcomes of critically } \\
\text { ill patients with } \\
\text { Covid-19 in Greece. }\end{array}$ & $\begin{array}{c}\text { Retrospective } \\
\text { multicenter } \\
\text { study, data and } \\
\text { outcomes of } 90 \\
\text { critically ill } \\
\text { patients } \\
\text { confirmed by } \\
\text { RNA 2109-nCoV } \\
\text { from } 8 \text { hospitals } \\
\text { across Greece } \\
\text { were analyzed. }\end{array}$ & 90 & $\begin{array}{l}72(80 \%) \\
\text { Masculine }\end{array}$ & 65 & $31(34,4 \%)$ & - & $\begin{array}{c}12(38,7 \%) \\
12 / 31\end{array}$ & $\begin{array}{c}\text { Obesity was more } \\
\text { frequent }(46.2 \%) \\
\text { among the deceased } \\
\text { than among the } \\
\text { survivors }(26.7 \%)(\mathrm{p}= \\
0.077)\end{array}$ & $\begin{array}{l}\text { The } p \text { value } \\
\text { suggests that there } \\
\text { may be a } \\
\text { relationship } \\
\text { between obesity } \\
\text { and mortality }\end{array}$ & $\begin{array}{l}\text { Retrospective data; } \\
\text { Absence of test results; }\end{array}$ \\
\hline 20 & $\begin{array}{c}\text { Features of } 20133 \\
\text { UK patients in } \\
\text { hospital with covid- } \\
19 \text { using the } \\
\text { ISARIC WHO } \\
\text { Clinical } \\
\text { Characterization } \\
\text { Protocol: } \\
\text { prospective } \\
\text { observational } \\
\text { cohort study. }\end{array}$ & $\begin{array}{l}\text { Docherty, } \\
\text { et al., } 2020\end{array}$ & $\begin{array}{l}\text { English } \\
\text { United } \\
\text { Kingdom }\end{array}$ & $\begin{array}{l}\text { Describe the clinical } \\
\text { characteristics of } \\
\text { inpatients with } 2019 \\
\text { coronavirus disease } \\
\text { (covid-19) in the UK }\end{array}$ & $\begin{array}{c}\text { Prospective } \\
\text { observational } \\
\text { cohort study with } \\
\text { rapid data } \\
\text { collection and } \\
\text { near real-time } \\
\text { analysis. }\end{array}$ & 20.133 & $\begin{array}{c}12068 \\
(59,9 \%) \\
\text { Masculine }\end{array}$ & 73 & $2.215(11 \%)$ & - & - & $\begin{array}{c}\text { Mortality } \\
\text { HR 1.33 [IC 95\% } \\
1.19-1.49] \\
\text { p }<0,001\end{array}$ & $\begin{array}{l}\text { Obesity was } \\
\text { associated with } \\
\text { higher hospital } \\
\text { mortality. }\end{array}$ & $\begin{array}{l}\text { Absence of records in } \\
\text { medical records; }\end{array}$ \\
\hline
\end{tabular}


Research, Society and Development, v. 10, n. 13, e165101321038, 2021

(CC BY 4.0) | ISSN 2525-3409 | DOI: http://dx.doi.org/10.33448/rsd-v10i13.21038

\begin{tabular}{|c|c|c|c|c|c|c|c|c|c|c|c|c|c|c|}
\hline 21 & $\begin{array}{l}\text { Features of patients } \\
\text { that died for } \\
\text { COVID-19 in a } \\
\text { hospital in the south } \\
\text { of Mexico: A } \\
\text { observational } \\
\text { cohort study. }\end{array}$ & $\begin{array}{c}\text { Ruíz- } \\
\text { Quiñonez } \\
\text { et al., } 2021\end{array}$ & $\begin{array}{l}\text { English } \\
\text { Mexico }\end{array}$ & $\begin{array}{c}\text { Evaluate } \\
\text { demographic } \\
\text { characteristics, } \\
\text { clinical } \\
\text { characteristics and } \\
\text { pharmacological } \\
\text { treatment of } \\
\text { individuals who died } \\
\text { from COVID-19 in } \\
\text { southern Mexico. }\end{array}$ & $\begin{array}{c}\text { Observational } \\
\text { study including } \\
\text { information from } \\
185 \text { deceased } \\
\text { people with a } \\
\text { confirmed } \\
\text { diagnosis of } \\
\text { COVID-19. Data } \\
\text { were retrieved } \\
\text { from medical } \\
\text { records. } \\
\end{array}$ & 185 & $\begin{array}{l}95(60,1 \%) \\
\text { Masculine }\end{array}$ & $\cong 59$ & $41(25,9 \%)$ & - & $\therefore$ & $\begin{array}{l}\text { Mortality } \\
\text { HR } 1.92\end{array}$ & $\begin{array}{l}\text { Epidemiological } \\
\text { data from COVID- } \\
19 \text { in Mexico } \\
\text { indicate that obesity } \\
\text { is a risk factor for } \\
\text { death and a partial } \\
\text { mediator of the } \\
\text { effects of diabetes } \\
\text { on reduced survival }\end{array}$ & $\begin{array}{l}\text { Absence of records in } \\
\text { medical records; }\end{array}$ \\
\hline 22 & $\begin{array}{l}\text { ICU outcomes in } \\
\text { Covid-19 patients } \\
\text { with obesity. }\end{array}$ & $\begin{array}{l}\text { Parikh et } \\
\text { al., } 2020\end{array}$ & $\begin{array}{l}\text { English } \\
\text { USA }\end{array}$ & $\begin{array}{l}\text { A study explores the } \\
\text { clinical relationship } \\
\text { between obesity and } \\
\text { Covid-19 in patients } \\
\text { admitted to an } \\
\text { intensive care unit } \\
\text { (ICU) in a tertiary } \\
\text { hospital. }\end{array}$ & $\begin{array}{l}\text { We conducted a } \\
\text { retrospective } \\
\text { cohort study of } \\
\text { adult patients } \\
\text { with laboratory- } \\
\text { confirmed severe } \\
\text { acute respiratory } \\
\text { syndrome } \\
\text { coronavirus 2 } \\
\end{array}$ & 160 & $\begin{array}{l}46(55,4 \%) \\
\text { Masculine }\end{array}$ & $\cong 56$ & $83(52 \%)$ & $\begin{array}{c}\text { IMV } \\
52(62,6 \%) \\
(52 / 83)\end{array}$ & $\begin{array}{c}26(31,3 \%) \\
(26 / 83)\end{array}$ & $\begin{array}{c}\text { IMV } \\
\text { OR: } 2.0[\text { [IC } 95 \% 1.1- \\
3.8]\end{array}$ & $\begin{array}{l}\text { Obese patients were } \\
\text { twice as likely to } \\
\text { need mechanical } \\
\text { ventilation. } \\
\text { However, there was } \\
\text { no significant } \\
\text { relationship } \\
\text { between obesity } \\
\text { and death. }\end{array}$ & $\begin{array}{l}\text { Small sample size; } \\
\text { Absence of records in } \\
\text { medical records; }\end{array}$ \\
\hline 23 & $\begin{array}{l}\text { Obesity and the } \\
\text { Risk of Intubation } \\
\text { or Death in Patients } \\
\text { With Coronavirus } \\
\text { Disease } 2019 .\end{array}$ & $\begin{array}{l}\text { Frank, et } \\
\text { al., } 2020\end{array}$ & $\begin{array}{l}\text { English } \\
\text { USA }\end{array}$ & $\begin{array}{l}\text { To characterize the } \\
\text { impact of obesity on } \\
\text { disease severity in } \\
\text { patients with } \\
\text { coronavirus disease. }\end{array}$ & $\begin{array}{l}\text { Retrospective } \\
\text { cohort study. }\end{array}$ & 305 & $\begin{array}{l}128(42 \%) \\
\text { Feminine }\end{array}$ & 60 & $\begin{array}{l}127 \\
(42 \%)\end{array}$ & - & - & $\begin{array}{c}\text { IMV } \\
\geq 30 \mathrm{~kg} / \mathrm{m} 2 \text { to }< \\
35 \mathrm{~kg} / \mathrm{m} 2 \\
\text { HR: } 2.1[\text { IC } 95 \% 1.2- \\
3.9] \\
\text { Mortality } \\
\text { BMI } \geq 35 \mathrm{~kg} / \mathrm{m} 2 \\
\text { HR: } 2.0[\text { [IC } 95 \% 1.1- \\
3.6] \\
\mathrm{p}=0.02\end{array}$ & $\begin{array}{l}\text { Obesity was an } \\
\text { independent risk } \\
\text { factor for IMV and } \\
\text { death. }\end{array}$ & $\begin{array}{l}\text { Small sample size; } \\
\text { Absence of BMI } \\
\text { registration; }\end{array}$ \\
\hline 24 & $\begin{array}{l}\text { Obesity is } \\
\text { Associated with } \\
\text { Increased Risk for } \\
\text { Mortality Among } \\
\text { Hospitalized } \\
\text { Patients with } \\
\text { COVID-19. }\end{array}$ & $\begin{array}{l}\text { Petti, et al., } \\
\quad 2020\end{array}$ & $\begin{array}{l}\text { English } \\
\text { USA }\end{array}$ & $\begin{array}{l}\text { Determining whether } \\
\text { obesity is a risk } \\
\text { factor for mortality } \\
\text { among patients with } \\
\text { COVID - } 19 .\end{array}$ & $\begin{array}{l}\text { Study was a } \\
\text { retrospective } \\
\text { cohort. }\end{array}$ & 238 & $\begin{array}{c}113(47,5 \%) \\
\text { Masculine }\end{array}$ & $\cong 58,5$ & $146(61,3 \%)$ & $\begin{array}{c}\text { ICU } \\
38(26 \%) \\
38 / 146 \\
\\
\text { IMV } \\
23 / 146 \\
(15,7 \%)\end{array}$ & $\begin{array}{l}14(9 \%) \\
(14 / 146)\end{array}$ & $\begin{array}{c}\text { Mortality } \\
\text { OR: } 1.7[\text { IC } 95 \% 1,1- \\
2,8] \\
p=0,016\end{array}$ & $\begin{array}{c}\text { Obesity was } \\
\text { considered a } \\
\text { significant predictor } \\
\text { of mortality among } \\
\text { hospitalized } \\
\text { patients with } \\
\text { COVID - } 19 \text { after } \\
\text { adjusting for age, } \\
\text { sex and other } \\
\text { comorbidities. } \\
\end{array}$ & $\begin{array}{c}\text { Small sample size and the } \\
\text { fact that it is a retrospective } \\
\text { analysis. }\end{array}$ \\
\hline 25 & $\begin{array}{l}\text { Obesity predisposes } \\
\text { to the risk of higher } \\
\text { mortality in young } \\
\text { COVID-19 patients. }\end{array}$ & $\begin{array}{l}\text { Zhang, et } \\
\text { al., 2020 }\end{array}$ & $\begin{array}{l}\text { English } \\
\text { China }\end{array}$ & $\begin{array}{c}\text { Check whether } \\
\text { obesity is a risk } \\
\text { factor associated with } \\
\text { death in young } \\
\text { patients }\end{array}$ & $\begin{array}{l}\text { Study was a } \\
\text { retrospective } \\
\text { cohort. }\end{array}$ & 53 & - & - & - & - & $\begin{array}{c}12(27,9 \%) \\
12 / 43\end{array}$ & $\begin{array}{c}\text { Mortality } \\
\text { OR: } 1,3[\text { [IC } 95 \%= \\
1,075-1,70] \\
p=0,010\end{array}$ & $\begin{array}{l}\text { Obesity was a risk } \\
\text { factor associated } \\
\text { with the occurrence } \\
\text { of mortality }\end{array}$ & $\begin{array}{l}\text { Limited number of sample } \\
\text { size }\end{array}$ \\
\hline 26 & $\begin{array}{l}\text { Obesity prolongs } \\
\text { the hospital stay in } \\
\text { patients affected by } \\
\text { COVID-19, and } \\
\text { may impact on } \\
\text { SARS-COV-2 } \\
\text { shedding. }\end{array}$ & $\begin{array}{l}\text { Moriconi, } \\
\text { et al., } 2020\end{array}$ & $\begin{array}{l}\text { English } \\
\text { Italy }\end{array}$ & $\begin{array}{l}\text { Investigate whether } \\
\text { obesity is a predictor } \\
\text { of worse outcome in } \\
\text { patients with } \\
\text { COVID. }\end{array}$ & $\begin{array}{c}\text { This } \\
\text { retrospective } \\
\text { observational } \\
\text { study was based } \\
\text { on medical } \\
\text { records of } \\
\text { hospitalized } \\
\text { patients. }\end{array}$ & 100 & $\begin{array}{l}57(57 \%) \\
\text { Masculine }\end{array}$ & 69 & $29(29 \%)$ & - & $\begin{array}{c}5(17,2 \%) \\
5 / 29\end{array}$ & - & $\begin{array}{c}\text { In partial least } \\
\text { squares regression, } \\
\text { there was no } \\
\text { statistical } \\
\text { association between } \\
\text { obesity and } \\
\text { mortality. }\end{array}$ & Sample size; \\
\hline
\end{tabular}


Research, Society and Development, v. 10, n. 13, e165101321038, 2021

(CC BY 4.0) | ISSN 2525-3409 | DOI: http://dx.doi.org/10.33448/rsd-v10i13.21038

\begin{tabular}{|c|c|c|c|c|c|c|c|c|c|c|c|c|c|c|}
\hline 27 & $\begin{array}{c}\text { Obesity and } \\
\text { smoking as risk } \\
\text { factors for invasive } \\
\text { mechanical } \\
\text { ventilation in } \\
\text { COVID-19: A } \\
\text { retrospective, } \\
\text { observational } \\
\text { cohort study. } \\
\end{array}$ & $\begin{array}{l}\text { Monteiro } \\
\text { et al., } 2020\end{array}$ & $\begin{array}{l}\text { English } \\
\text { USA }\end{array}$ & $\begin{array}{l}\text { Describe the } \\
\text { trajectory of } \\
\text { respiratory failure in } \\
\text { COVID-19 and } \\
\text { explore factors } \\
\text { associated with the } \\
\text { risk of invasive } \\
\text { mechanical } \\
\text { ventilation (IMV). } \\
\end{array}$ & $\begin{array}{c}\text { Retrospective } \\
\text { and observational } \\
\text { cohort study with } \\
\text { electronic } \\
\text { medical records } \\
\text { of hospitalized } \\
\text { adult patients. }\end{array}$ & 112 & $\begin{array}{l}74(66 \%) \\
\text { Masculine }\end{array}$ & 61 & $40(36 \%)$ & $\begin{array}{l}\text { IMV } \\
17 / 40 \\
(42,5 \%)\end{array}$ & - & $\begin{array}{c}\text { IMV } \\
\text { OR } 5.82[\text { IC } 1,74- \\
19,48] \\
p=0,004\end{array}$ & $\begin{array}{l}\text { Obesity was } \\
\text { associated with a } \\
\text { greater need for } \\
\text { IMV among } \\
\text { hospitalized } \\
\text { patients. }\end{array}$ & - \\
\hline 28 & $\begin{array}{l}\text { Outcomes for } \\
\text { patients with } \\
\text { COVID-19 } \\
\text { admitted to } \\
\text { Australian intensive } \\
\text { care units during } \\
\text { the first four } \\
\text { months of the } \\
\text { pandemic. }\end{array}$ & $\begin{array}{l}\text { Burell et } \\
\text { al., } 2020\end{array}$ & $\begin{array}{c}\text { English } \\
\text { Australia }\end{array}$ & $\begin{array}{l}\text { To describe the } \\
\text { characteristics and } \\
\text { outcomes of patients } \\
\text { with COVID-19 } \\
\text { admitted to intensive } \\
\text { care units (ICUs) } \\
\text { during the first } \\
\text { months of the } \\
\text { pandemic in } \\
\text { Australia. }\end{array}$ & $\begin{array}{l}\text { Prospective } \\
\text { observational } \\
\text { cohort study in } \\
77 \text { ICUs across } \\
\text { Australia. }\end{array}$ & 204 & $\begin{array}{l}140(69 \%) \\
\text { Masculine }\end{array}$ & 63 & $81(40 \%)$ & $\begin{array}{c}\text { IMV } \\
52 / 81 \\
(64,1 \%)\end{array}$ & $\begin{array}{c}8(9,8 \%) \\
8 / 29\end{array}$ & $\begin{array}{c}\text { Death } \\
\text { OR } 0,32 \text { [IC } 0,13- \\
0,79]\end{array}$ & $\begin{array}{l}\text { There was no } \\
\text { statistical } \\
\text { correlation between } \\
\text { death and mortality. }\end{array}$ & $\begin{array}{l}\text { Non-exploitation of } \\
\text { decision-making regarding } \\
\text { admission to the ICU. And } \\
\text { the observational nature of } \\
\text { the study. }\end{array}$ \\
\hline 29 & $\begin{array}{c}\text { Relationship } \\
\text { between obesity } \\
\text { and severe COVID- } \\
19 \text { outcomes in } \\
\text { patients with type } 2 \\
\text { diabetes: Results } \\
\text { from the } \\
\text { CORONADO } \\
\text { study. } \\
\end{array}$ & $\begin{array}{l}\text { Smati et } \\
\text { al., } 2021\end{array}$ & $\begin{array}{l}\text { English } \\
\text { France }\end{array}$ & $\begin{array}{l}\text { To assess the } \\
\text { relationship between } \\
\text { body mass index } \\
\text { (BMI) classes and } \\
\text { COVID-19 early } \\
\text { prognosis in } \\
\text { hospitalized patients } \\
\text { with type } 2 \text { diabetes } \\
\text { (DM2). }\end{array}$ & $\begin{array}{c}\text { From the } \\
\text { CORONAvirus- } \\
\text { SARS-CoV-2 } \\
\text { and Diabetes } \\
\text { Outcomes } \\
\text { (CORONADO) } \\
\text { study, an analysis } \\
\text { was performed in } \\
\text { patients with } \\
\text { DM2. }\end{array}$ & 1965 & $\begin{array}{l}1267(64 \%) \\
\text { Masculine }\end{array}$ & 71 & $805(41 \%)$ & $\begin{array}{c}\text { IMV } \\
195(24 \%) \\
195 / 805\end{array}$ & $\begin{array}{c}78(9,6 \%) \\
78 / 805\end{array}$ & $\begin{array}{c}\text { IMV } \\
\text { Class II: } 1,94 \text { [IC } \\
\text { 1,36-2,76], } \mathrm{p}=0,0003 \\
\text { Class III: 2,63 [IC,81- } \\
\text { 3,83], p }<0,0001\end{array}$ & $\begin{array}{c}\text { There was an } \\
\text { association between } \\
\text { obesity and } \\
\text { intubation, but there } \\
\text { was no statistical } \\
\text { association with } \\
\text { mortality. }\end{array}$ & $\begin{array}{l}\text { The specificity of patients: } \\
\text { hospitalized with DM2; } \\
\text { Short follow-up period; }\end{array}$ \\
\hline 30 & $\begin{array}{c}\text { Risk factors for } \\
\text { mortality in } \\
\text { hospitalized } \\
\text { patients with } \\
\text { COVID-19 at the } \\
\text { start of the } \\
\text { pandemic in } \\
\text { Belgium: a } \\
\text { retrospective cohort } \\
\text { study. } \\
\end{array}$ & $\begin{array}{l}\text { Van Halem } \\
\text { et al., } 2020\end{array}$ & $\begin{array}{l}\text { English } \\
\text { Belgium }\end{array}$ & $\begin{array}{l}\text { Identify the main risk } \\
\text { factors for hospital } \\
\text { mortality of patients } \\
\text { with COVID. }\end{array}$ & $\begin{array}{l}\text { Retrospective } \\
\text { cohort study, } \\
\text { with data } \\
\text { collection } \\
\text { through } \\
\text { electronic } \\
\text { medical records. }\end{array}$ & 319 & $\begin{array}{l}191(60 \%) \\
\text { Masculine }\end{array}$ & 74 & $\begin{array}{c}54(23 \%) \\
54 / 230\end{array}$ & - & $\begin{array}{c}19(35,1 \%) \\
19 / 54\end{array}$ & - & $\begin{array}{l}\text { In the univariate } \\
\text { analysis, patients } \\
\text { with obesity had an } \\
\text { overall increased } \\
\text { risk of death, while } \\
\text { overweight, on the } \\
\text { other hand, showed } \\
\text { a trend toward } \\
\text { lower mortality. }\end{array}$ & $\begin{array}{l}\text { The retrospective cohort } \\
\text { was carried out in a single } \\
\text { center; } \\
\text { There was no correction for } \\
\text { multiple comparisons, } \\
\text { making predictors with p } \\
\text { values close to } 0.05 \text { less } \\
\text { likely to be true } \\
\text { associations. }\end{array}$ \\
\hline 31 & $\begin{array}{c}\text { Selective CD8 cell } \\
\text { reduction by SARS- } \\
\text { CoV-2 is associated } \\
\text { with a worse } \\
\text { prognosis and } \\
\text { systemic } \\
\text { inflammation in } \\
\text { COVID-19 patients. }\end{array}$ & $\begin{array}{l}\text { Urra et al., } \\
2020\end{array}$ & $\begin{array}{l}\text { English } \\
\text { Spain }\end{array}$ & $\begin{array}{c}\text { To analyze } \\
\text { comorbidities, } \\
\text { inflammation } \\
\text { indicators such as } \\
\text { CRP and the } \\
\text { neutrophil/lymphocyt } \\
\text { e ratio, as well as the } \\
\text { count of blood cells } \\
\text { with T lymphocyte } \\
\text { subtypes in patients } \\
\text { hospitalized with } \\
\text { Pneumonia COVID- } \\
19 .\end{array}$ & $\begin{array}{l}\text { A retrospective } \\
\text { case-control } \\
\text { study was carried } \\
\text { out in patients } \\
\text { with COVID-19 } \\
\text { pneumonia } \\
\text { admitted to the } \\
\text { University } \\
\text { Hospital of } \\
\text { Ciudad Real } \\
\text { (Spain) }\end{array}$ & 172 & $\begin{array}{l}104(60 \%) \\
\text { Masculine }\end{array}$ & - & $17(9 \%)$ & $\begin{array}{c}\text { ICU } \\
7 / 17(25 \%)\end{array}$ & - & $\begin{array}{c}\text { ICU } \\
\text { OR }=4,72[\text { IC 95\% } \\
1,614-13,830, p= \\
0,005]\end{array}$ & $\begin{array}{c}\text { There was a } \\
\text { statistical } \\
\text { association between } \\
\text { obesity and } \\
\text { admission to the } \\
\text { ICU }\end{array}$ & - \\
\hline
\end{tabular}


Research, Society and Development, v. 10, n. 13, e165101321038, 2021

(CC BY 4.0) | ISSN 2525-3409 | DOI: http://dx.doi.org/10.33448/rsd-v10i13.21038

\begin{tabular}{|c|c|c|c|c|c|c|c|c|c|c|c|c|c|c|}
\hline 32 & $\begin{array}{c}\text { Severe Obesity as } \\
\text { an Independent } \\
\text { Risk Factor for } \\
\text { COVID-19 } \\
\text { Mortality in } \\
\text { Hospitalized } \\
\text { Patients Younger } \\
\text { than } 50 .\end{array}$ & $\begin{array}{l}\text { Klang et } \\
\text { al., } 2020\end{array}$ & $\begin{array}{l}\text { English } \\
\text { USA }\end{array}$ & $\begin{array}{l}\text { To analyze obesity as } \\
\text { an independent risk } \\
\text { factor for mortality in } \\
\text { hospitalized patients } \\
\text { under } 50 \text { years of } \\
\text { age. }\end{array}$ & $\begin{array}{c}\text { We } \\
\text { retrospectively } \\
\text { analyzed data } \\
\text { from patients } \\
\text { with COVID-19 } \\
\text { hospitalized in a } \\
\text { large academic } \\
\text { hospital system } \\
\text { in New York } \\
\text { City. }\end{array}$ & 3406 & $\begin{array}{c}1961 \\
(57,5 \%) \\
\text { Masculine }\end{array}$ & - & $1231(36 \%)$ & - & - & $\begin{array}{c}\leq 50 \text { anos: } \text { BMI }>40 \\
\quad(\mathrm{OR}=5,1) ; \\
>50 \text { anos: } \mathrm{BMI}>40 \\
\quad(\mathrm{OR}=1,6) ;\end{array}$ & $\begin{array}{c}\text { Hospitalized } \\
\text { patients under } 50 \\
\text { years of age with } \\
\text { severe obesity are } \\
\text { more likely to die } \\
\text { from COVID-19. }\end{array}$ & $\begin{array}{l}\text { Retrospective study; small } \\
\text { number of patients under } \\
50 \text { years old }\end{array}$ \\
\hline 33 & $\begin{array}{c}\text { Severe obesity, } \\
\text { increasing age and } \\
\text { male sex are } \\
\text { independently } \\
\text { associated with } \\
\text { worse in-hospital } \\
\text { outcomes, and } \\
\text { higher in-hospital } \\
\text { mortality, in a } \\
\text { cohort of patients } \\
\text { with COVID-19 in } \\
\text { the Bronx, New } \\
\text { York. }\end{array}$ & $\begin{array}{l}\text { Palaiodimo } \\
\text { s et al., } \\
2020\end{array}$ & $\begin{array}{l}\text { English } \\
\text { USA }\end{array}$ & $\begin{array}{l}\text { Evaluate the } \\
\text { characteristics and } \\
\text { baseline outcomes of } \\
\text { patients hospitalized } \\
\text { with COVID-19 in } \\
\text { the Bronx and } \\
\text { investigate whether } \\
\text { obesity is associated } \\
\text { with worse outcomes } \\
\text { regardless of age, } \\
\text { sex, and other } \\
\text { comorbidities. }\end{array}$ & $\begin{array}{l}\text { Retrospective } \\
\text { study with } \\
\text { review of } \\
\text { electronic } \\
\text { medical records, }\end{array}$ & 200 & $\begin{array}{l}102(51 \%) \\
\text { Feminine }\end{array}$ & 64 & $162(81 \%)$ & $\begin{array}{c}\text { IMV } \\
35(21,6 \%) \\
35 / 162 \\
\\
\text { ICU } \\
29(17,9 \%) \\
29 / 162\end{array}$ & $\begin{array}{c}36(22 \%) \\
36 / 162\end{array}$ & $\begin{array}{c}\text { Mortality } \\
\text { BMI } \geq 35 \mathrm{~kg} / \mathrm{m}^{2}: \text { OR } \\
3,78[\text { [IC } 95 \%: 1,45- \\
9,83] \mathrm{p}=0,006)\end{array}$ & $\begin{array}{l}\text { Severe obesity was } \\
\text { associated with } \\
\text { higher hospital } \\
\text { mortality, even } \\
\text { after adjusting for } \\
\text { other pertinent } \\
\text { potentially } \\
\text { confounding } \\
\text { factors. }\end{array}$ & $\begin{array}{l}\text { Retrospective study and } \\
\text { reduced sample size }\end{array}$ \\
\hline 34 & $\begin{array}{l}\text { The impact of } \\
\text { obesity on } \\
\text { COVID-19 } \\
\text { complications: a } \\
\text { retrospective cohort } \\
\text { study. }\end{array}$ & $\begin{array}{l}\text { Nakeshban } \\
\text { di et al., } \\
2020\end{array}$ & $\begin{array}{l}\text { English } \\
\text { USA }\end{array}$ & $\begin{array}{l}\text { To elucidate the } \\
\text { association between } \\
\text { obesity and COVID- } \\
19 \text { outcomes. }\end{array}$ & $\begin{array}{c}\text { Retrospective } \\
\text { cohort study of } \\
\text { patients } \\
\text { hospitalized with } \\
\text { COVID-19. }\end{array}$ & 504 & $\begin{array}{l}263(52 \%) \\
\text { Masculine }\end{array}$ & 68 & $215(43 \%)$ & $\begin{array}{l}\text { IMV } \\
60(28 \%) \\
60 / 215\end{array}$ & $\begin{array}{c}87(42,4 \%) \\
87 / 215\end{array}$ & $\begin{array}{c}\text { Mortality } \\
\text { RR } 1,3[\text { IC } 95 \% 1,0- \\
1,7] \mathrm{p}=0,04 \\
\text { IMV } \\
\text { RR 2,4 [IC } 95 \% 1,5- \\
4,0] \mathrm{p}=0,001\end{array}$ & $\begin{array}{c}\text { Overweight and } \\
\text { obese patients who } \\
\text { have COVID-19 are } \\
\text { at increased risk of } \\
\text { mortality and } \\
\text { intubation } \\
\text { compared to those } \\
\text { with normal BMI. }\end{array}$ & $\begin{array}{l}\text { Retrospective observational } \\
\text { study, and presence of } \\
\text { confounding bias for some } \\
\text { outcomes. }\end{array}$ \\
\hline 35 & $\begin{array}{l}\text { The importance of } \\
\text { overweight in } \\
\text { COVID-19: A } \\
\text { retrospective } \\
\text { analysis in a single } \\
\text { center of Wuhan, } \\
\text { China. }\end{array}$ & $\begin{array}{l}\text { Rao et al., } \\
2020\end{array}$ & $\begin{array}{c}\text { English } \\
\text { China }\end{array}$ & $\begin{array}{c}\text { Evaluate the } \\
\text { association between } \\
\text { overweight and } \\
\text { severity, drug } \\
\text { response and clinical } \\
\text { outcomes of the new } \\
\text { coronavirus 2019 } \\
\text { disease (COVID-19). }\end{array}$ & $\begin{array}{l}\text { Retrospective } \\
\text { cohort study, } \\
\text { with review of } \\
\text { medical records. }\end{array}$ & 240 & $\begin{array}{c}129(53,8 \%) \\
\text { Feminine }\end{array}$ & 48 & $114(47,5 \%)$ & $\begin{array}{c}\text { Severe } \\
\text { Pneumonia } \\
73(64 \%) \\
73 / 114\end{array}$ & - & $\begin{array}{c}\text { Severe Pneumonia } \\
\text { OR 3,075 [IC 95\% } \\
1,18-7,96]), p=0,021\end{array}$ & $\begin{array}{l}\text { Overweight is } \\
\text { associated with a } \\
\text { severe form of } \\
\text { pneumonia, but it is } \\
\text { not associated with } \\
\text { death. }\end{array}$ & $\begin{array}{l}\text { Descriptive study with } \\
\text { reduced sample size. }\end{array}$ \\
\hline 36 & $\begin{array}{c}\text { The obesity } \\
\text { paradox: Analysis } \\
\text { from the SMAtteo } \\
\text { COvid-19 REgistry } \\
\text { (SMACORE) } \\
\text { cohort. }\end{array}$ & $\begin{array}{l}\text { Biscarini et } \\
\text { al., } 2020\end{array}$ & $\begin{array}{l}\text { English } \\
\text { Italy }\end{array}$ & $\begin{array}{l}\text { To examine the } \\
\text { correlation between } \\
\text { obesity, 30-day } \\
\text { mortality and } \\
\text { admission to the } \\
\text { Intensive Care Unit } \\
\text { (ICU) in patients } \\
\text { with Covid-19. }\end{array}$ & $\begin{array}{c}\text { Retrospective } \\
\text { cohort study with } \\
\text { revised medical } \\
\text { records and } \\
\text { analysis of } \\
\text { demographic, } \\
\text { clinical, and } \\
\text { outcome data. }\end{array}$ & 427 & $\begin{array}{l}291(68,1 \%) \\
\text { Masculine }\end{array}$ & 67 & $\begin{array}{l}74 / 331 \\
(22,3 \%)\end{array}$ & $\begin{array}{c}\text { ICU } \\
21(28,4 \%) \\
21 / 74\end{array}$ & $\begin{array}{c}25(33,8 \%) \\
25 / 74\end{array}$ & $\begin{array}{c}\text { ICU } \\
\text { OR 1,96 [IC 95\% } \\
1,03-3,75] p=0,001\end{array}$ & $\begin{array}{l}\text { Obesity was } \\
\text { associated with ICU } \\
\text { admission, but it } \\
\text { was not associated } \\
\text { with death. }\end{array}$ & $\begin{array}{l}\text { Descriptive study, with } \\
\text { reduced sample size, and } \\
\text { non-differentiation of BMI } \\
\text { calculation between } \\
\text { adipose and muscle tissue. }\end{array}$ \\
\hline
\end{tabular}


Research, Society and Development, v. 10, n. 13, e165101321038, 2021

(CC BY 4.0) | ISSN 2525-3409 | DOI: http://dx.doi.org/10.33448/rsd-v10i13.21038

\begin{tabular}{|c|c|c|c|c|c|c|c|c|c|c|c|c|c|c|}
\hline 37 & $\begin{array}{l}\text { The relationship } \\
\text { between obesity, } \\
\text { hemoglobin A1c } \\
\text { and the severity of } \\
\text { COVID-19 at an } \\
\text { urban tertiary care } \\
\text { center in New York } \\
\text { City: a retrospective } \\
\text { cohort study. }\end{array}$ & $\begin{array}{l}\text { Randhawa } \\
\text { et al., } 2021\end{array}$ & $\begin{array}{l}\text { English } \\
\text { USA }\end{array}$ & $\begin{array}{c}\text { Determine whether } \\
\text { obesity and diabetes } \\
\text { are risk factors for } \\
\text { serious outcomes in } \\
\text { COVID-19 and } \\
\text { compare patient } \\
\text { outcomes in these } \\
\text { two conditions. }\end{array}$ & $\begin{array}{l}\text { Retrospective } \\
\text { cohort study. }\end{array}$ & 302 & $\begin{array}{c}195(64,5 \%) \\
\text { Masculine }\end{array}$ & - & $152(50,3 \%)$ & $\begin{array}{c}\text { IMV } \\
71(46,7 \%) \\
71 / 152\end{array}$ & - & $\begin{array}{c}\text { Patients with obesity } \\
\text { and/or diabetes were } \\
\text { more likely to develop } \\
\text { ARDS (79 patients x } \\
57 \text { patients, } \mathrm{p}< \\
0.0001) \text { and to be } \\
\text { IMV (71 patients } \mathrm{x} 45 \\
\text { patients, } \mathrm{p}=0.0031)\end{array}$ & $\begin{array}{c}\text { Patients with } \\
\text { obesity and/or } \\
\text { diabetes were more } \\
\text { likely to experience } \\
\text { serious } \\
\text { complications but } \\
\text { had insignificant } \\
\text { differences in } \\
\text { mortality }\end{array}$ & $\begin{array}{l}\text { Absence of blood gas data; } \\
\text { Distribution of body fat in } \\
\text { the elderly. }\end{array}$ \\
\hline 38 & $\begin{array}{l}\text { The role of obesity } \\
\text { in inflammatory } \\
\text { markers in COVID- } \\
19 \text { patients. }\end{array}$ & $\begin{array}{l}\text { McNeill et } \\
\text { al., } 2021\end{array}$ & $\begin{array}{l}\text { English } \\
\text { USA }\end{array}$ & $\begin{array}{c}\text { Evaluate } \\
\text { inflammatory } \\
\text { markers in obese and } \\
\text { non-obese } \\
\text { individuals } \\
\text { hospitalized for } \\
\text { COVID-19. } \\
\end{array}$ & $\begin{array}{l}\text { Retrospective } \\
\text { cohort study. }\end{array}$ & 781 & $\begin{array}{l}453(58 \%) \\
\text { Masculine }\end{array}$ & 61 & $349(45 \%)$ & $\begin{array}{c}\text { ICU } \\
129(37 \%) \\
129 / 349 \\
\\
\text { IMV } \\
109(31 \%) \\
109 / 349 \\
\end{array}$ & $\begin{array}{c}49(14 \%) \\
49 / 349\end{array}$ & $\begin{array}{c}\text { Mortality } \\
\text { OR 2,20 [IC 95\% } \\
1,31-3,70] p=0,003)\end{array}$ & $\begin{array}{l}\text { Obesity was } \\
\text { associated with } \\
\text { mortality }\end{array}$ & $\begin{array}{l}\text { There may have been an } \\
\text { investigation bias, as } \\
\text { inflammatory markers were } \\
\text { not measured at defined } \\
\text { intervals. In addition, the } \\
\text { fact that it is a single-center } \\
\text { research. } \\
\end{array}$ \\
\hline 39 & $\begin{array}{l}\text { The swedish covid- } \\
19 \text { intensive care } \\
\text { cohort: Risk factors } \\
\text { of ICU admission } \\
\text { and ICU mortality. }\end{array}$ & $\begin{array}{l}\text { Ahlström } \\
\text { et al., } 2021\end{array}$ & $\begin{array}{l}\text { English } \\
\text { Sweden }\end{array}$ & $\begin{array}{c}\text { To assess the impact } \\
\text { of pre-COVID-19 } \\
\text { medications, the } \\
\text { comorbidity-related } \\
\text { risk for ICU } \\
\text { admission as an } \\
\text { indicator of severe } \\
\text { disease in COVID- } \\
19 \text {, and the effect of } \\
\text { these factors on the } \\
\text { risk of mortality in } \\
\text { the ICU. }\end{array}$ & $\begin{array}{l}\text { Retrospective } \\
\text { study of data } \\
\text { analysis of } \\
\text { patients admitted } \\
\text { to the ICU }\end{array}$ & 1981 & $\begin{array}{l}1465(74 \%) \\
\text { Masculine }\end{array}$ & 61 & $123(6,2 \%)$ & - & $\begin{array}{c}19(15,4 \%) \\
19 / 123\end{array}$ & $\begin{array}{c}\text { ICU } \\
\text { OR 2,37 [IC 95\% } \\
1,81-3,11] p=0,001\end{array}$ & $\begin{array}{l}\text { Obesity was related } \\
\text { to admission to the } \\
\text { ICU, but it was not } \\
\text { related to death } \\
\text { from COVID-19. }\end{array}$ & $\begin{array}{l}\text { Patients who were referred } \\
\text { to the ICU, but who were } \\
\text { not admitted due to wear } \\
\text { and tear on the healthcare } \\
\text { system. }\end{array}$ \\
\hline
\end{tabular}

Source: Research Protocol (2020) 
Among the observed data shown in the table above, there is a prevalence of males (77\%) with an average age of 61 years, demonstrating that this is an elderly population. When evaluating the incidence of obese patients hospitalized by Covid-19, the mean was 33\%, compared to obese patients who required Invasive Mechanical Ventilation (IMV) it was approximately $37 \%$.

\section{Discussion}

The surveys carried out in the USA corresponded to $37.5 \%$ (15) of the studies included in this review, while 35.89\% (14) studies were carried out in European countries. Although Vasconcelos et al., (2020) demonstrate that China stood out in scientific production related to COVID-19, presenting the largest number of publications related to the topic in 2019 and 2020 , the leading role of obesity-related research in the US may be a reflection of the high incidence of this comorbidity, being present in 35\% of the North American population, as described by De Sousa et al., (2008). Thus, there was greater concern about the implications of the correlation between obesity and the occurrence of COVID-19 in the context of the pandemic.

Regarding the adopted methodology, $89.7 \%$ (35) of the studies were carried out through retrospective data review. According to Fernandes et al, (2005), among the weaknesses/limitations of this type of study there is the information bias and the inability to control confounding variables (lack of information), this fact corroborates the data collected from the studies in this review where the potential or the real lack of information was one of the points most evident as limitations in the studies, along with the sample size and the difficulty of measuring outcomes in a pandemic scenario.

There was great variation in the number of patients in the populations studied, with samples ranging from 53 to 89,450 participants, with an average of 3864 participants per survey. Regarding the characteristics of the population, the average age was 61 years among the studies, this information was not found in 04 studies. The prevalence of older adults in the samples is evident, which according to the authors may also have corroborated for the outcomes. Matsudo et al, 2000 says that there is evidence in the literature that aging brings neuromotor, metabolic and anthropometric variations to the population.

In the studies analyzed, there was a prevalence of males in 77\% (30) of the researches, only 01 article did not take gender into account. This is consistent with some studies that confirmed that men have characteristics that enhance the disease, due to greater disregard for health and low use of primary and secondary care, being at greater risk of clinical decompensation (Camarano, 2002; Almeida et al., 2015).

For the definition of obesity, the classification proposed by the WHO was used, in which the BMI that determines obesity is above $30 \mathrm{~kg} / \mathrm{m} 3$. It was possible to identify the information on the prevalence of obesity in 37 studies, 01 there was no information on the number of obese patients in relation to the population studied, and in another study, the BMI value $>24 \mathrm{~kg} / \mathrm{m}^{2}$ was considered for statistical analysis purposes. That would fit the overweight classification, according to the WHO. The percentage incidence of obese patients hospitalized by Covid in the extracted data ranged from $0.7 \%$ to $81 \%$, with a mean of 33\%. Even before the new coronavirus pandemic, obesity was already considered a global challenge, however, with the restrictions established by governments and health promotion/protection entities that aimed to reduce the circulation of the virus, there was na increase in the sedentary and weight gain (Posso et al., 2020).

Insulin resistance, changes in metabolism, hyperglycemia, and increased inflammatory markers are just some of the changes Obesity itself is a metabolic entity characterized by changes related to its occurrence. This, combined with vascular and pulmonary alterations, as well as the increased expression of the angiotensin 2 converting enzyme, and the adipose tissue itself, which can behave as a means for intense viral replication, thus creating na exacerbated immune response, make this comorbidity na important predictive and prognostic factor for understanding the course of the disease and its severity (Saryer et al., 2020; Guzik et al., 2020; Shah et al., 2021). 
Of the evaluated articles, 23 brought data from obese patients who had severe complications, some of them evaluating more than one outcome: invasive mechanical ventilation (IMV) (n 9) and/or admission to the intensive care unit (n 9) and/or occurrence of severe pneumonia (n 2), or only outcomes defined as severe (n 3). The percentage of obese hospitalized patients who required IMV ranged from 15.7 to $64.1 \%$, with na average of approximately $37 \%$, while the percentage of obese patients with covid who were admitted to the ICU ranged from $17.9 \%$ to 62 , 5\% with na average of $41 \%$. And the relationship between obesity and mortality was assessed in 24 articles, with a death rate ranging from $9 \%$ to $65.7 \%$, with a mean death rate of $29.7 \%$. It is worth noting that among the articles mentioned, there were 02 (two) studies that showed the relationship between obesity and the IMV and mortality outcomes, stratifying by degree of obesity according to the WHO classification, in these it was shown that the greater the degree of obesity, the higher the percentage of patients who evolve with IMV and death.

Regarding statistical associations (odds ratio, relative risk and hazard ratio), 06 (six) studies evaluated the correlation between obesity and IMV in $100 \%$ of them there was a statistical correlation, which showed that obese patients are 2 to 5 times more likely to need VMI. When the outcome evaluated was admission to the ICU, in all 06 (six) articles that evaluated this outcome, there was a positive correlation, as the occurrence of obesity makes the patient with COVID 1.5 to 4.7 times more likely to be admitted to the intensive care unit. In one of the studies where there was stratification by race, only obese blacks were associated with intensive care. The occurrence of death in obese patients was evaluated in 29 articles, of which 17 (58.6\%) showed a significant correlation between obesity and increased mortality, one of the studies showed na 8 fold increase in the chances of death in obese patients.

Some studies report that when considering a scenario of mechanical ventilation, obese patients would be subject to longer periods of intubation, greater difficulty in weaning from mechanical ventilation and handling by professionals in nonspecialized health centers, factors that could contribute for unfavorable outcomes (Severin et al., 2020; Stefan et al., 2020). Muscogiuri et al., (2020), points out that patients with COVID with a BMI value above $30 \mathrm{~kg} / \mathrm{m}^{2}$ and who were referred for mechanical ventilation had a mortality rate of $60.9 \%$. Furthermore, it was also observed that the recommendation and need for mechanical ventilation increased with the degree of obesity, which may explain the variability between studies. In addition, it is difficult to obtain the patients' BMI data, since most of the time this value is obtained in na estimated way during hospitalization.

In the study by Thomsom et al. (2020), the absence and unclear criteria for admission to the intensive care units (ICU) would be a confounding factor in the analysis of admitted patients. In this regard, some scores created to assess hospitalized patients with COVID are already available, which aim to identify those who need intensive support in less time, helping clinical decision-making in a stressful and low-resource context (Zhao et al., 2020; Kumar et al., 2020)

Louie et al. (2011) and Dawood et al. (2009) reported during the H1N1 influenza epidemic a large proportion of hospital admissions and mortality were associated with obesity, and now a growing number of studies suggest that the outcomes of obese patients are worse compared to non-obese patients. Obese individuals who became infected with the coronavirus. It is a fact that mortality from COVID has multifactorial causes, among which we can highlight: diabetes, cardiovascular, cerebrovascular, pulmonary diseases and advanced age are some of the predictors already identified (Muscogiuri et al., 2020; Li et al., 2020; Du et al., 2020; Kang et al., 2020; Ruan et al., 2020; Grasselli et al., 2020).

\section{Conclusion}

According to the studies analyzed, they showed that obesity increases the incidence of unfavorable outcomes, such as increased ICU admission, increased intubation and invasive mechanical ventilation, that is, obesity is a risk factor for the development of the severe form of the disease. The findings of this study also concluded that mortality was associated in 50\% 
of the studies. It is important to mention that obesity is usually associated with several other pathologies, such as: hypertension, diabetes and cardiovascular diseases, which are also a risk factor for the development of the severe form of COVID -19. Therefore, there is a need for studies that seek to correlate to which dangers obese individuals and those with comorbidities are exposed.

\section{References}

Abohamr, S. I., Abazid, R. M., Aldossari, M. A., Amer, H. A., Badhawi, O. S., Aljunaidi, O. M., Alzarzour, S. H., Saadeddin, H. M., Bhat, F. A., \& Elsheikh, E. (2020). Clinical characteristics and in-hospital mortality of COVID-19 adult patients in Saudi Arabia. Saudi medical journal, 41(11), 1217-1226. https://doi.org/10.15537/smj.2020.11.25495

Ahlström, B., Frithiof, R., Hultström, M., Larsson, I. M., Strandberg, G., \& Lipcsey, M. (2021). The swedish covid-19 intensive care cohort: Risk factors of ICU admission and ICU mortality. Acta anaesthesiologica Scandinavica, 65(4), 525-533. Advance online publication. https://doi.org/10.1111/aas.13781.

Almeida, A. V., Tavares Mafra, S. C., da Silva, E. P., \& Kanso, S. (2015). A Feminização da Velhice: em foco as características socioeconômicas, pessoais e familiares das idosas e o risco social / The Feminization of Old Age: a focus on the socioeconomic, personal and family characteristics of the elderly and the social risk. Textos \& Contextos (Porto Alegre), 14(1), 115 - 131. https://doi.org/10.15448/1677-9509.2015.1.19830.

Biscarini, S., Colaneri, M., Ludovisi, S., Seminari, E., Pieri, T. C., Valsecchi, P., Gallazzi, I., Giusti, E., Cammà, C., Zuccaro, V., \& Mondelli, M. U. (2020). The obesity paradox: Analysis from the SMAtteo COvid-19 REgistry (SMACORE) cohort. Nutrition, metabolism, and cardiovascular diseases: NMCD, 30(11), 1920-1925. https://doi.org/10.1016/j.numecd.2020.07.047.

BRASIL, Ministério da Saúde. Painel Coronavirus Brasil. https://covid.saude.gov.br/.

BRASIL. Ministério da Saúde. Portaria No 188 de 03 de fevereiro de 2020. Declara emergência em Saúde Pública de importância Nacional (ESPIN) em decorrência da Infecção Humana pelo novo Coronavírus (2019-nCoV). Disponível em: https://www.in.gov.br/web/dou/-/portaria-n-188-de-3-de-fevereirode-2020-241408388. Acessado em: 15/10/2020

Burrell, A. J., Pellegrini, B., Salimi, F., Begum, H., Broadley, T., Campbell, L. T., Cheng, A. C., Cheung, W., Cooper, D. J., Earnest, A., Erickson, S. J., French, C. J., Kaldor, J. M., Litton, E., Murthy, S., McAllister, R. E., Nichol, A. D., Palermo, A., Plummer, M. P., Ramanan, M., \& Udy, A. A. (2021). Outcomes for patients with COVID-19 admitted to Australian intensive care units during the first four months of the pandemic. The Medical journal of Australia, 214(1), 23-30. https://doi.org/10.5694/mja2.50883.

Camarano, A. A. (2002). Envelhecimento da população brasileira: uma contribuição demográfica. In: Freitas EV et al, organizadores. Tratado de Geriatria e Gerontologia. Rio de Janeiro (RJ):Guanabara Koogan.

Castro, H. M., Canale, H. L., Ferreyro, B. L., Prieto, M. A., Massimino, B. E., Funtowicz, G., \& Maritano Furcada, J. (2020). Características clínicas de la enfermedad por Coronavirus 2019 en un centro de Argentina. Cohorte retrospective. MEDICINA (Buenos Aires), 80, 35-43.

COVID-ICU Group on behalf of the REVA Network and the COVID-ICU Investigators (2021). Clinical characteristics and day-90 outcomes of 4244 critically ill adults with COVID-19: a prospective cohort study. Intensive care medicine, 47(1), 60-73. https://doi.org/10.1007/s00134-020-06294-x.

Dawood, F. S., Iuliano, A. D., Reed, C., Meltzer, M. I., Shay, D. K., Cheng, P. Y., Bandaranayake, D., Breiman, R. F., Brooks, W. A., Buchy, P., Feikin, D. R., Fowler, K. B., Gordon, A., Hien, N. T., Horby, P., Huang, Q. S., Katz, M. A., Krishnan, A., Lal, R., Montgomery, J. M., \& Widdowson, M. A. (2012). Estimated global mortality associated with the first 12 months of 2009 pandemic influenza A H1N1 virus circulation: a modelling study. The Lancet. Infectious diseases, 12(9), 687-695. https://doi.org/10.1016/S1473-3099(12)70121-4.

De Andrade, C., Pereira, C., Martins, M., Lima, S., \& Portela, M. C. (2020). COVID-19 hospitalizations in Brazil's Unified Health System (SUS). PloS one, 15(12), e0243126. https://doi.org/10.1371/journal.pone.0243126.

De Paiva, A. M., da Silva Pinto, A. W., Cançado, B. L., Chequer, F. M. D., Pereira, M. L., \& Baldoni, A. O. (2020). Efeito das “promessas terapêuticas” sobre os preços de medicamentos em tempos de pandemia. Journal of Health \& Biological Sciences, 8(1), 1-5. http://dx.doi.org/10.12662/23173076jhbs.v8i1.3407.p1-5.2020.

De Sousa, A. G. P., Cercato, C., Mancini, M. C., \& Halpern, A. (2008). Obesity and obstructive sleep apnea-hypopnea syndrome. Obesity Reviews, 9(4), 340354. https://doi.org/10.1111/j.1467-789X.2008.00478.x.

Dixon, A. E., \& Peters, U. (2018). The effect of obesity on lung function. Expert review of respiratory medicine, 12(9), 755-767. https://doi.org/10.1080/17476348.2018.1506331.

Docherty, A. B., Harrison, E. M., Green, C. A., Hardwick, H. E., Pius, R., Norman, L., Holden, K. A., Read, J. M., Dondelinger, F., Carson, G., Merson, L., Lee, J., Plotkin, D., Sigfrid, L., Halpin, S., Jackson, C., Gamble, C., Horby, P. W., Nguyen-Van-Tam, J. S., Ho, A., \& ISARIC4C investigators (2020). Features of 20133 UK patients in hospital with covid-19 using the ISARIC WHO Clinical Characterisation Protocol: prospective observational cohort study. BMJ (Clinical research ed.), 369, m1985. https://doi.org/10.1136/bmj.m1985.

Du, R. H., Liang, L. R., Yang, C. Q., Wang, W., Cao, T. Z., Li, M., Guo, G. Y., Du, J., Zheng, C. L., Zhu, Q., Hu, M., Li, X. Y., Peng, P., \& Shi, H. Z. (2020). Predictors of mortality for patients with COVID-19 pneumonia caused by SARS-CoV-2: a prospective cohort study. The European respiratory journal, 55(5), 2000524. https://doi.org/10.1183/13993003.00524-2020.

Fernandes, S. M., \& Carneiro, A. V. (2005). Tipos de estudos clínicos. II. Estudos de coorte. Revista portuguesa de cardiologia, $24(9)$, 1151-58. 
Filardo, T. D., Khan, M. R., Krawczyk, N., Galitzer, H., Karmen-Tuohy, S., Coffee, M., Schaye, V. E., Eckhardt, B. J., \& Cohen, G. M. (2020). Comorbidity and clinical factors associated with COVID-19 critical illness and mortality at a large public hospital in New York City in the early phase of the pandemic (March-April 2020). PloS one, 15(11), e0242760. https://doi.org/10.1371/journal.pone.0242760.

Frank, R. C., Mendez, S. R., Stevenson, E. K., Guseh, J. S., Chung, M., \& Silverman, M. G. (2020). Obesity and the Risk of Intubation or Death in Patients With Coronavirus Disease 2019. Critical care medicine, 48(11), e1097-e1101. https://doi.org/10.1097/CCM.0000000000004553.

Giacomelli, A., Ridolfo, A. L., Milazzo, L., Oreni, L., Bernacchia, D., Siano, M., Bonazzetti, C., Covizzi, A., Schiuma, M., Passerini, M., Piscaglia, M., Coen, M., Gubertini, G., Rizzardini, G., Cogliati, C., Brambilla, A. M., Colombo, R., Castelli, A., Rech, R., Riva, A., \& Galli, M. (2020). 30-day mortality in patients hospitalized with COVID-19 during the first wave of the Italian epidemic: A prospective cohort study. Pharmacological research, $158,104931$. https://doi.org/10.1016/j.phrs.2020.104931.

Grasselli, G., Zangrillo, A., Zanella, A., Antonelli, M., Cabrini, L., Castelli, A., Cereda, D., Coluccello, A., Foti, G., Fumagalli, R., Iotti, G., Latronico, N., Lorini, L., Merler, S., Natalini, G., Piatti, A., Ranieri, M. V., Scandroglio, A. M., Storti, E., Cecconi, M., ... COVID-19 Lombardy ICU Network (2020). Baseline Characteristics and Outcomes of 1591 Patients Infected With SARS-CoV-2 Admitted to ICUs of the Lombardy Region, Italy. JAMA, 323(16), 15741581. https://doi.org/10.1001/jama.2020.5394.

Gregoriano, C., Koch, D., Haubitz, S., Conen, A., Fux, C. A., Mueller, B., Bernasconi, L., Hammerer-Lercher, A., Oberle, M., Burgermeister, S., Reiter, H., Kutz, A., \& Schuetz, P. (2020). Characteristics, predictors and outcomes among 99 patients hospitalised with COVID-19 in a tertiary care centre in Switzerland: an observational analysis. Swiss medical weekly, 150, w20316. https://doi.org/10.4414/smw.2020.20316.

Guzik, T. J., Mohiddin, S. A., Dimarco, A., Patel, V., Savvatis, K., Marelli-Berg, F. M., Madhur, M. S., Tomaszewski, M., Maffia, P., D'Acquisto, F., Nicklin, S. A., Marian, A. J., Nosalski, R., Murray, E. C., Guzik, B., Berry, C., Touyz, R. M., Kreutz, R., Wang, D. W., Bhella, D., \& McInnes, I. B. (2020). COVID19 and the cardiovascular system: implications for risk assessment, diagnosis, and treatment options. Cardiovascular research, $116(10)$, 1666-1687. https://doi.org/10.1093/cvr/cvaa106.

Halvatsiotis, P., Kotanidou, A., Tzannis, K., Jahaj, E., Magira, E., Theodorakopoulou, M., Konstandopoulou, G., Gkeka, E., Pourzitaki, C., Kapravelos, N., Papoti, S., Sileli, M., Gogos, C., Velissaris, D., Markou, N., Stefanatou, E., Vlachogianni, G., Aimoniotou, E., Komnos, A., Zafeiridis, T., \& Dimopoulos, G. (2020). Demographic and clinical features of critically ill patients with COVID-19 in Greece: The burden of diabetes and obesity. Diabetes research and clinical practice, 166, 108331. https://doi.org/10.1016/j.diabres.2020.108331.

Hendren, N. S., de Lemos, J. A., Ayers, C., Das, S. R., Rao, A., Carter, S., Rosenblatt, A., Walchok, J., Omar, W., Khera, R., Hegde, A. A., Drazner, M. H., Neeland, I. J., \& Grodin, J. L. (2021). Association of Body Mass Index and Age With Morbidity and Mortality in Patients Hospitalized With COVID-19: Results From the American Heart Association COVID-19 Cardiovascular Disease Registry. Circulation, 143(2), 135-144. https://doi.org/10.1161/CIRCULATIONAHA.120.051936.

Holanda M.A., Pinheiro B.V. (2020). COVID-19 pandemic and mechanical ventilation: facing the present, designing the future. Journal Brasileira de Pneumologia,46(4):e20200282

Hu, B., Zeng, L. P., Yang, X. L., Ge, X. Y., Zhang, W., Li, B., Xie, J. Z., Shen, X. R., Zhang, Y. Z., Wang, N., Luo, D. S., Zheng, X. S., Wang, M. N., Daszak, P., Wang, L. F., Cui, J., \& Shi, Z. L. (2017). Discovery of a rich gene pool of bat SARS-related coronaviruses provides new insights into the origin of SARS coronavirus. PLoS pathogens, 13(11), e1006698. https://doi.org/10.1371/journal.ppat.1006698.

Kaeuffer, C., Le Hyaric, C., Fabacher, T., Mootien, J., Dervieux, B., Ruch, Y., Hugerot, A., Zhu, Y. J., Pointurier, V., Clere-Jehl, R., Greigert, V., Kassegne, L., Lefebvre, N., Gallais, F., Covid Alsace Study Group, Meyer, N., Hansmann, Y., Hinschberger, O., Danion, F., \& COVID Alsace Study Group (2020). Clinical characteristics and risk factors associated with severe COVID-19: prospective analysis of 1,045 hospitalised cases in North-Eastern France, March 2020. Euro surveillance: bulletin Europeen sur les maladies transmissibles = European communicable disease bulletin, 25(48), 2000895. https://doi.org/10.2807/1560-7917.ES.2020.25.48.2000895.

Kang Y. J. (2020). Mortality Rate of Infection With COVID-19 in Korea From the Perspective of Underlying Disease. Disaster medicine and public health preparedness, 14(3), 384-386. https://doi.org/10.1017/dmp.2020.60.

Kim, T. S., Roslin, M., Wang, J. J., Kane, J., Hirsch, J. S., Kim, E. J., \& Northwell Health COVID-19 Research Consortium (2021). BMI as a Risk Factor for Clinical Outcomes in Patients Hospitalized with COVID-19 in New York. Obesity (Silver Spring, Md.), 29(2), 279-284. https://doi.org/10.1002/oby.23076.

Klang, E., Kassim, G., Soffer, S., Freeman, R., Levin, M. A., \& Reich, D. L. (2020). Severe Obesity as an Independent Risk Factor for COVID-19 Mortality in Hospitalized Patients Younger than 50. Obesity (Silver Spring, Md.), 28(9), 1595-1599. https://doi.org/10.1002/oby.22913.

Kolinski, J. M., Sundararajan, S. K., Swartz, S., Naik, K. V., \& Gupta, N. (2020). COVID-19 Experience in a Wisconsin Academic Medical Center. WMJ: official publication of the State Medical Society of Wisconsin, 119(3), 198-201.

Kulcsar, K. A., Coleman, C. M., Beck, S. E., \& Frieman, M. B. (2019). Comorbid diabetes results in immune dysregulation and enhanced disease severity following MERS-CoV infection. JCI insight, 4(20), e131774. https://doi.org/10.1172/jci.insight.131774.

Kumar, A., Kumar, N., Kumar, A., \& Kumar, A. (2020). COVID-19 pandemic and the need for objective criteria for ICU admissions. Journal of clinical anesthesia, 66, 109945. https://doi.org/10.1016/j.jclinane.2020.109945.

Li, X., Xu, S., Yu, M., Wang, K., Tao, Y., Zhou, Y., Shi, J., Zhou, M., Wu, B., Yang, Z., Zhang, C., Yue, J., Zhang, Z., Renz, H., Liu, X., Xie, J., Xie, M., \& Zhao, J. (2020). Risk factors for severity and mortality in adult COVID-19 inpatients in Wuhan. The Journal of allergy and clinical immunology, 146(1), 110 118. https://doi.org/10.1016/j.jaci.2020.04.006.

Louie, J. K., Acosta, M., Samuel, M. C., Schechter, R., Vugia, D. J., Harriman, K., Matyas, B. T., \& California Pandemic (H1N1) Working Group (2011). A novel risk factor for a novel virus: obesity and 2009 pandemic influenza A (H1N1). Clinical infectious diseases: an official publication of the Infectious Diseases Society of America, 52(3), 301-312. https://doi.org/10.1093/cid/ciq152. 
Matsudo, S. M., Matsudo, V. K. R., \& Barros Neto, T. L. (2000). Impacto do envelhecimento nas variáveis antropométricas, neuromotoras e metabólicas da aptidão física. Revista brasileira de ciência e movimento, 8(4), 21-32.

McNeill, J. N., Lau, E. S., Paniagua, S. M., Liu, E. E., Wang, J. K., Bassett, I. V., Selvaggi, C. A., Lubitz, S. A., Foulkes, A. S., \& Ho, J. E. (2021). The role of obesity in inflammatory markers in COVID-19 patients. Obesity research \& clinical practice, 15(1), 96-99. https://doi.org/10.1016/j.orcp.2020.12.004.

Monteiro, A. C., Suri, R., Emeruwa, I. O., Stretch, R. J., Cortes-Lopez, R. Y., Sherman, A., Lindsay, C. C., Fulcher, J. A., Goodman-Meza, D., Sapru, A., Buhr, R. G., Chang, S. Y., Wang, T., \& Qadir, N. (2020). Obesity and smoking as risk factors for invasive mechanical ventilation in COVID-19: A retrospective, observational cohort study. PloS one, 15(12), e0238552. https://doi.org/10.1371/journal.pone.0238552.

Moriconi, D., Masi, S., Rebelos, E., Virdis, A., Manca, M. L., De Marco, S., Taddei, S., \& Nannipieri, M. (2020). Obesity prolongs the hospital stay in patients affected by COVID-19, and may impact on SARS-COV-2 shedding. Obesity research \& clinical practice, 14(3), 205-209. https://doi.org/10.1016/j.orcp.2020.05.009.

Mostaghim, A., Sinha, P., Bielick, C., Knudsen, S., Beeram, I., White, L. F., Apovian, C., Sagar, M., \& Hochberg, N. S. (2020). Clinical outcomes and inflammatory marker levels in patients with Covid-19 and obesity at an inner-city safety net hospital. PloS one, 15(12), e0243888. https://doi.org/10.1371/journal.pone.0243888.

Muscogiuri, G., Pugliese, G., Barrea, L., Savastano, S., \& Colao, A. (2020). Commentary: Obesity: The "Achilles heel" for COVID-19? Metabolism: clinical and experimental, 108, 154251. https://doi.org/10.1016/j.metabol.2020.154251.

Nachega, J. B., Ishoso, D. K., Otokoye, J. O., Hermans, M. P., Machekano, R. N., Sam-Agudu, N. A., Bongo-Pasi Nswe, C., Mbala-Kingebeni, P., Madinga, J. N., Mukendi, S., Kolié, M. C., Nkwembe, E. N., Mbuyi, G. M., Nsio, J. M., Mukeba Tshialala, D., Tshiasuma Pipo, M., Ahuka-Mundeke, S., MuyembeTamfum, J. J., Mofenson, L., Smith, G., ... Kayembe, J. M. (2020). Clinical Characteristics and Outcomes of Patients Hospitalized for COVID-19 in Africa: Early Insights from the Democratic Republic of the Congo. The American journal of tropical medicine and hygiene, 103(6), 2419-2428. https://doi.org/10.4269/ajtmh.20-1240.

Nakeshbandi, M., Maini, R., Daniel, P., Rosengarten, S., Parmar, P., Wilson, C., Kim, J. M., Oommen, A., Mecklenburg, M., Salvani, J., Joseph, M. A., \& Breitman, I. (2020). The impact of obesity on COVID-19 complications: a retrospective cohort study. International journal of obesity (2005), 44(9), 18321837. https://doi.org/10.1038/s41366-020-0648-x.

Oliveira, M. A. P., \& Parente, R. C. M. (2010). Estudos de coorte e de caso-controle na era da medicina baseada em evidência. Brazilian Journal of Videoendoscopic Surgery, 3(3), 115-25.

Organização Mundial da Saude e Organização Pan-Americana de Saúde. Folha Informativa Covid-19. Disponível em: https://www.paho.org/pt/covid19. Acessado em: 18/10/2020

Palaiodimos, L., Kokkinidis, D. G., Li, W., Karamanis, D., Ognibene, J., Arora, S., Southern, W. N., \& Mantzoros, C. S. (2020). Severe obesity, increasing age and male sex are independently associated with worse in-hospital outcomes, and higher in-hospital mortality, in a cohort of patients with COVID-19 in the Bronx, New York. Metabolism: clinical and experimental, 108, 154262. https://doi.org/10.1016/j.metabol.2020.154262.

Parikh, R., Garcia, M. A., Rajendran, I., Johnson, S., Mesfin, N., Weinberg, J., \& Reardon, C. C. (2020). ICU outcomes in Covid-19 patients with obesity. Therapeutic advances in respiratory disease, 14, 1753466620971146. https://doi.org/10.1177/1753466620971146.

Peng, Y., Meng, K., He, M., Zhu, R., Guan, H., Ke, Z., Leng, L., Wang, X., Liu, B., Hu, C., Ji, Q., Keerman, M., Cheng, L., Wu, T., Huang, K., \& Zeng, Q. (2020). Clinical Characteristics and Prognosis of 244 Cardiovascular Patients Suffering From Coronavirus Disease in Wuhan, China. Journal of the American Heart Association, 9(19), e016796. https://doi.org/10.1161/JAHA.120.016796.

Petrou, Christos. Guest Post - Scientific output in the year of COVID. The Scholarly Kitchen, 19, 2020. https://scholarlykitchen.sspnet.org/2020/11/19/guestpost-scientific-output-in-the-year-of-covid/.

Pettit, N. N., MacKenzie, E. L., Ridgway, J. P., Pursell, K., Ash, D., Patel, B., \& Pho, M. T. (2020). Obesity is Associated with Increased Risk for Mortality Among Hospitalized Patients with COVID-19. Obesity (Silver Spring, Md.), 28(10), 1806-1810. https://doi.org/10.1002/oby.22941.

Posso, M., Comas, M., Román, M., Domingo, L., Louro, J., González, C., Sala, M., Anglès, A., Cirera, I., Cots, F., Frías, V. M., Gea, J., Güerri-Fernández, R., Masclans, J. R., Noguès, X., Vázquez, O., Villar-García, J., Horcajada, J. P., Pascual, J., \& Castells, X. (2020). Comorbidities and Mortality in Patients With COVID-19 Aged 60 Years and Older in a University Hospital in Spain. Archivos de bronconeumologia, 56(11), 756-758. https://doi.org/10.1016/j.arbres.2020.06.012.

Randhawa, G., Syed, K. A., Singh, K., Kundal, S. V., Oli, S., Silver, M., Syed, S. A., Suban Na Ayutthaya, T., Williams, S., Lodato, Z. L., Rozvadovskiy, V., Kamholz, S., \& Wolf, L. (2021). The relationship between obesity, hemoglobin A1c and the severity of COVID-19 at an urban tertiary care center in New York City: a retrospective cohort study. BMJ open, 11(1), e044526. https://doi.org/10.1136/bmjopen-2020-044526.

Rao, X., Wu, C., Wang, S., Tong, S., Wang, G., Wu, G., \& Zhou, R. (2020). The importance of overweight in COVID-19: A retrospective analysis in a single center of Wuhan, China. Medicine, 99(43), e22766. https://doi.org/10.1097/MD.0000000000022766.

Ruan, Q., Yang, K., Wang, W., Jiang, L., \& Song, J. (2020). Correction to: Clinical predictors of mortality due to COVID-19 based on an analysis of data of 150 patients from Wuhan, China. Intensive care medicine, 46(6), 1294-1297. https://doi.org/10.1007/s00134-020-06028-z.

Ruíz-Quiñonez, J. A., Guzmán-Priego, C. G., Nolasco-Rosales, G. A., Tovilla-Zarate, C. A., Flores-Barrientos, O. I., Narváez-Osorio, V., Baeza-Flores, G., Gonzalez-Castro, T. B., López-Brito, C. R., Denis-García, C. A., Pérez-García, A., \& Juárez-Rojop, I. E. (2021). Features of patients that died for COVID19 in a hospital in the south of Mexico: A observational cohort study. PloS one, 16(2), e0245394. https://doi.org/10.1371/journal.pone.0245394.

Saraiva, E. M. S., Ricarte, É. C., Coelho, J. L. G., de Sousa, D. F., da Silva Feitosa, F. L., Alves, R. S., ... \& de Santana, W. J. (2020). Impacto da pandemia pelo Covid-19 na provisão de equipamentos de proteção individual. Brazilian Journal of Development, 6(7), 43751-43762. https://doi.org/10.34117/bjdv6n7-115. 
Sarver, D. C., \& Wong, G. W. (2021). Obesity alters Ace2 and Tmprss2 expression in lung, trachea, and esophagus in a sex-dependent manner: Implications for COVID-19. Biochemical and biophysical research communications, 538, 92-96. https://doi.org/10.1016/j.bbrc.2020.10.066.

Severin, R., Arena, R., Lavie, C. J., Bond, S., \& Phillips, S. A. (2020). Respiratory Muscle Performance Screening for Infectious Disease Management Following COVID-19: A Highly Pressurized Situation. The American journal of medicine, 133(9), 1025-1032. https://doi.org/10.1016/j.amjmed.2020.04.003.

Shah, H., Khan, M., Dhurandhar, N. V., \& Hegde, V. (2021). The triumvirate: why hypertension, obesity, and diabetes are risk factors for adverse effects in patients with COVID-19. Acta diabetologica, 58(7), 831-843. https://doi.org/10.1007/s00592-020-01636-z.

Smati, S., Tramunt, B., Wargny, M., Caussy, C., Gaborit, B., Vatier, C., Vergès, B., Ancelle, D., Amadou, C., Bachir, L. A., Bourron, O., Coffin-Boutreux, C., Barraud, S., Dorange, A., Fremy, B., Gautier, J. F., Germain, N., Larger, E., Laugier-Robiolle, S., Meyer, L., CORONADO investigators (2021). Relationship between obesity and severe COVID-19 outcomes in patients with type 2 diabetes: Results from the CORONADO study. Diabetes, obesity \& metabolism, 23(2), 391-403. https://doi.org/10.1111/dom.14228.

Stefan, N., Birkenfeld, A. L., Schulze, M. B., \& Ludwig, D. S. (2020). Obesity and impaired metabolic health in patients with COVID-19. Nature reviews. Endocrinology, 16(7), 341-342. https://doi.org/10.1038/s41574-020-0364-6.

Thomson, R. J., Hunter, J., Dutton, J., Schneider, J., Khosravi, M., Casement, A., Dhadwal, K., \& Martin, D. (2020). Clinical characteristics and outcomes of critically ill patients with COVID-19 admitted to an intensive care unit in London: A prospective observational cohort study. PloS one, 15(12), e0243710. https://doi.org/10.1371/journal.pone.0243710.

UNICAMP - Universidade Estadual de Campinas. Web Site: Coronavírusc Covid-19. Quanto custa? unicamp.br/unicamp/coronavirus/quanto_custa.

Urra, J. M., Cabrera, C. M., Porras, L., \& Ródenas, I. (2020). Selective CD8 cell reduction by SARS-CoV-2 is associated with a worse prognosis and systemic inflammation in COVID-19 patients. Clinical immunology (Orlando, Fla.), 217, 108486. https://doi.org/10.1016/j.clim.2020.108486.

van Halem, K., Bruyndonckx, R., van der Hilst, J., Cox, J., Driesen, P., Opsomer, M., Van Steenkiste, E., Stessel, B., Dubois, J., \& Messiaen, P. (2020). Risk factors for mortality in hospitalized patients with COVID-19 at the start of the pandemic in Belgium: a retrospective cohort study. BMC infectious diseases, 20(1), 897. https://doi.org/10.1186/s12879-020-05605-3.

Vasconcelos, I. G., \& do Nascimento, D. A. (2020). Mapeamento da produção científica sobre COVID-19. Interamerican Journal Of Medicine And Health, 3:e202003044. https://doi.org/10.31005/iajmh.v3i0.134

Wang, C., Horby, P. W., Hayden, F. G., \& Gao, G. F. (2020). A novel coronavirus outbreak of global health concern. Lancet (London, England), 395(10223), 470-473. https://doi.org/10.1016/S0140-6736(20)30185-9.

Wang, W., Tang, J., \& Wei, F. (2020). Updated understanding of the outbreak of 2019 novel coronavirus (2019-nCoV) in Wuhan, China. Journal of medical virology, 92(4), 441-447. https://doi.org/10.1002/jmv.25689.

Yoshida, Y., Gillet, S. A., Brown, M. I., Zu, Y., Wilson, S. M., Ahmed, S. J., Tirumalasetty, S., Lovre, D., Krousel-Wood, M., Denson, J. L., \& MauvaisJarvis, F. (2021). Clinical characteristics and outcomes in women and men hospitalized for coronavirus disease 2019 in New Orleans. Biology of sex differences, 12(1), 20. https://doi.org/10.1186/s13293-021-00359-2.

Zavaleta, M. H., Copaja Corzo, C. A., Bardales Silva, F., Flores Palacios, R., Barreto Rocchetti, L., \& Benites Zapata, V. A. (2021). Characteristics and risk factors for mortality in patients hospitalized by COVID-19 in a public hospital in Tacna. In Characteristics and risk factors for mortality in patients hospitalized by COVID-19 in a public hospital in Tacna. https://doi.org/10.1590/SciELOPreprints.1764.

Zhang, F., Xiong, Y., Wei, Y., Hu, Y., Wang, F., Li, G., Liu, K., Du, R., Wang, C. Y., \& Zhu, W. (2020). Obesity predisposes to the risk of higher mortality in young COVID-19 patients. Journal of medical virology, 92(11), 2536-2542. https://doi.org/10.1002/jmv.26039.

Zhao, Z., Chen, A., Hou, W., Graham, J. M., Li, H., Richman, P. S., Thode, H. C., Singer, A. J., \& Duong, T. Q. (2020). Prediction model and risk scores of ICU admission and mortality in COVID-19. PloS one, 15(7), e0236618. https://doi.org/10.1371/journal.pone.0236618. 